\title{
High-Fidelity Simulation of Pathogen Propagation, Transmission and Mitigation in the Built Environment
}

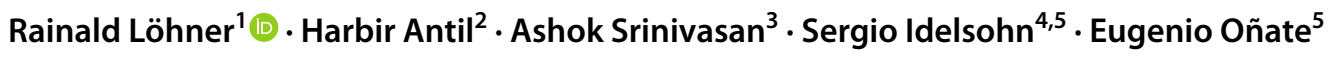

Received: 15 March 2021 / Accepted: 8 May 2021 / Published online: 5 July 2021

(c) CIMNE, Barcelona, Spain 2021

\begin{abstract}
An overview of high-fidelity modeling of pathogen propagation, transmission and mitigation in the built environment is given. In order to derive the required physical and numerical models, the current understanding of pathogen, and in particular virus transmission and mitigation is summarized. The ordinary and partial differential equations that describe the flow, the particles and possibly the UV radiation loads in rooms or HVAC ducts are presented, as well as proper numerical methods to solve them in an expedient way. Thereafter, the motion of pedestrians, as well as proper ways to couple computational fluid dynamics and computational crowd dynamics to enable high-fidelity pathogen transmission and infection simulations is treated. The present review shows that high-fidelity simulations of pathogen propagation, transmission and mitigation in the built environment have reached a high degree of sophistication, offering a quantum leap in accuracy from simpler probabilistic models. This is particularly the case when considering the propagation of pathogens via aerosols in the presence of moving pedestrians.
\end{abstract}

\section{Introduction}

Rainald Löhner

rlohner@gmu.edu

Harbir Antil

hantil@gmu.edu

Ashok Srinivasan

asrinivasan@uwf.edu

Sergio Idelsohn

sergio@cimne.upc.edu

Eugenio Oñate

onate@cimne.upc.edu

1 Center for Computational Fluid Dynamics, College of Science, George Mason University, Fairfax, VA 22030-4444, USA

2 Center for Mathematics and Artificial Intelligence, College of Science, George Mason University, Fairfax, VA 22030-4444, USA

3 Department of Computer Science, University of West Florida, Pensacola, FL 32514, USA

4 Catalan Institution for Research and Advanced Studies, ICREA, Barcelona, Spain

5 International Center for Numerical Methods in Engineering, CIMNE, Barcelona, Spain
Starting in Wuhan, China, in the fall of 2019, the Covid-19 pandemic has claimed and will continue to claim millions of infected patients (with as yet unknown long-term effects even after recovery) and hundreds of thousands of deaths. The lockdowns that followed this outbreak have led to mass unemployment, stalled economic development and loss of productivity that will take years to recover. Some changes in habits and lifestyles may be permanent: in the future, working from home or in a 'socially distanced manner' may be the prevalent modus operandi for large segments of society.

A positive effect of the pandemic has been the renewed interest in pathogen propagation, transmission and mitigation [23, 31, 32, 46-48, 113]. In particular, the relative impact of transmission via 'large droplets' versus 'small droplets' or aerosols has been debated throughout 2020 . This in turn has lead to a vigorous effort to model all of the phenomena associated with pathogen propagation, transmission and mitigation via advanced computational techniques-from the molecular scale to the scale of the built environment [1, 21, 22, 68, 79, 80, 117, 118].

Before describing the numerical and computational models and methodologies, a quick overview of pathogen, and in particular virus transmission and mitigation is given. This defines the relevant physical phenomena, which in turn 
define the ordinary and partial differential equations that describe the flow, the particles and possibly the UV radiation loads in rooms or heating, ventilation and air conditioning (HVAC) ducts. Thereafter, the models used for pedestrian motion are outlined. The sections on numerical methodologies conclude with a description of the coupling methodology employed to combine computational fluid dynamics (CFD) and computational crowd dynamics (CCD).

The combination of CFD and CCD is an enabling technology for high-fidelity pathogen transmission and infection simulations. Two models of varying complexity and sophistication are described.

Several examples, ranging from sneezing simulations in an aircraft cabin, a subway car and a classroom, to pathogen transmission simulations in train stations illustrate the applicability of the techniques developed.

\section{Virus Infection}

Before describing the modeling techniques for pathogen propagation, transmission and mitigation, a brief description of virus propagation and lifetime is given. Viruses are usually present in the air or some surface, and make their way into the body either via inhalation (nose, mouth), ingestion (mouth) or attachment (eyes, hands, clothes). In many cases the victim inadvertently touches an infected surface or viruses are deposited on its hands, and then the hands or clothes touch either the nose, the eyes or the mouth, thus allowing the virus to enter the body.
An open question of great importance is how many viruses it takes to overwhelm the body's natural defense mechanism and trigger an infection. This number, the socalled infectious dose, could depend on numerous factors, among them the state of immune defenses of the individual, the timing of viral entry (all at once, piece by piece), and the amount of hair and mucous in the nasal vessels [10]. In principle, a single organism in a favourable environment may replicate sufficiently to cause disease [99]. Data from research performed on biological warfare agents [27] suggests that both bacteria and viruses can produce disease with as few as 1-100 organisms (e.g. brucellosis 10-100, Q fever $1-10$, tularaemia $10-50$, smallpox $10-100$, viral haemorrhagic fevers 1-10 organisms, tuberculosis 1). Compare these numbers and consider that as many as 3000 organisms can be produced by talking for 5 min or a single cough, with sneezing producing many more $[69,84,85,104,111]$. Figure 1, reproduced from [104], shows a typical number and size distribution. It should be pointed out that this is a histogram of the relative counts of different particle sizes. There is often a large variation about the mean, which can impact disease risk estimates.

\subsection{Virus Lifetime Outside the Body}

Current evidence for Covid-19 points to lifetimes outside the body that can range from 1 to $2 \mathrm{~h}$ in air to several days on particular surfaces (so-called fomite transmission mode) $[49,60,107]$. There has also been some documentation of lifetime variation depending on humidity.
Fig. 1 Counts of particles of various diameters in air expelled by 90 coughs $[84]$

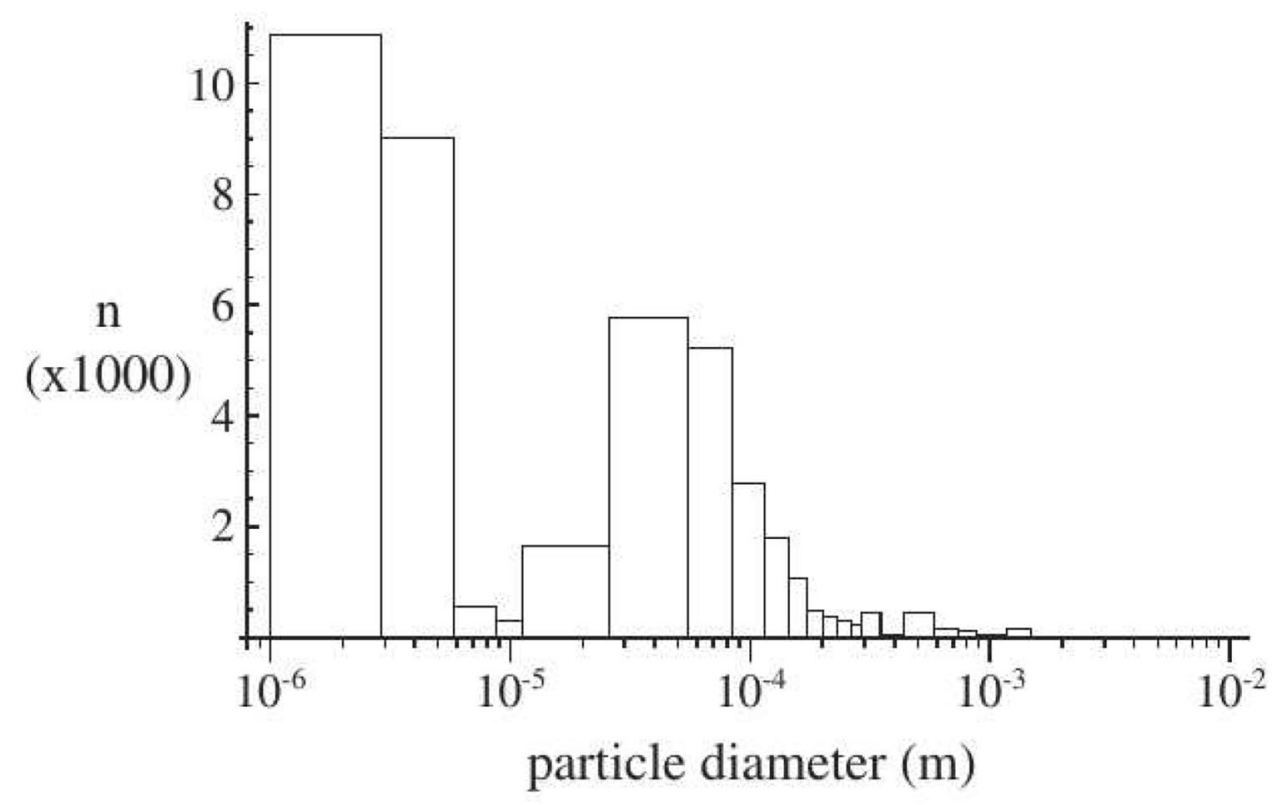




\subsection{Virus Transmission}

\subsubsection{Sneezing and Coughing}

In the sequel, we consider sneezing and coughing as the main conduits of pathogen transmission. Clearly, breathing and talking will lead to the exhalation of air, and, consequently the exhalation of viruses from infected victims [3-5]. However, it stands to reason that the size and amount of particles released - and hence the amount of viruses in them-is much higher and much more concentrated when sneezing or coughing $[3,5,25,59,70,104]$.

The velocity of air at a person's mouth during sneezing and coughing has been a source of heated debate, particularly in the media. The experimental evidence points to exit velocities of the order of $2-14 \mathrm{~m} / \mathrm{s}[33,34,101,102]$. A typical amount and size of particles can be seen in Fig. 1.

\subsubsection{Sink Velocities}

Table 1 lists the terminal sink velocities for water droplets in air based on the diameter [18, 95]. One can see that below diameters of $O(0.1 \mathrm{~mm})$ the sink velocity is very low, implying that these particles remain in and move with the air for considerable time (and possibly distances).

Table 1 Sink velocities and reynolds number for water particles in air

\begin{tabular}{lll}
\hline Diameter $(\mathrm{mm})$ & Sink velocity $(\mathrm{m} / \mathrm{s})$ & $\operatorname{Re}$ \\
\hline $1.00 \mathrm{E}-01$ & $3.01 \mathrm{E}-01$ & $1.99 \mathrm{E}+00$ \\
$1.00 \mathrm{E}-02$ & $3.01 \mathrm{E}-03$ & $1.99 \mathrm{E}-03$ \\
$1.00 \mathrm{E}-03$ & $3.01 \mathrm{E}-05$ & $1.99 \mathrm{E}-06$ \\
$1.00 \mathrm{E}-04$ & $3.01 \mathrm{E}-07$ & $1.99 \mathrm{E}-09$ \\
\hline
\end{tabular}

\subsubsection{Evaporation}

Depending on the relative humidity and the temperature of the ambient air, the smaller particles can evaporate in milliseconds. However, as the mucous and saliva evaporate, they build a gel-like structure that surrounds the virus, allowing it to survive. This implies that extremely small particles with possible viruses will remain infectious for extended periods of times - up to an hour according to some studies $[60,107]$.

An important question is whether a particle/droplet will first reach the ground or evaporate. Figure 2, taken from [114], shows that below $0.12 \mathrm{~mm}$ the particles evaporate before falling $2 \mathrm{~m}$ (i.e. reaching the ground).

\section{Mitigation Techniques}

In order to develop pathogen mitigation or elimination strategies, it is important to visualize the movement of the droplets by which they are carried:

- Larger $(1 \mathrm{~mm} \geq d \geq 0.1 \mathrm{~mm})$ droplets follow a ballistic path, are not significantly slowed down by the surrounding air, and drop and attach to the floor or any surface on the way in a time of approximately $O(1)$ s without considerable evaporation; visualize or conceptualize these as: spitting saliva;

- Smaller $(d<O(0.01) \mathrm{mm})$ droplets are immediately slowed down by the surrounding air, evaporate in a fraction of a second, and are being transported by the air; the best way to conceptualize these aerosolized droplets is as (invisible) cigarette smoke or sprays (e.g. hair spray or deodorants).
Fig. 2 Evaporation time and falling time of droplets of varying diameter $\left(T_{p 0}=33^{\circ} \mathrm{C}, T_{\infty}=18^{\circ} \mathrm{C}, R H=0 \%\right)$ From [114]

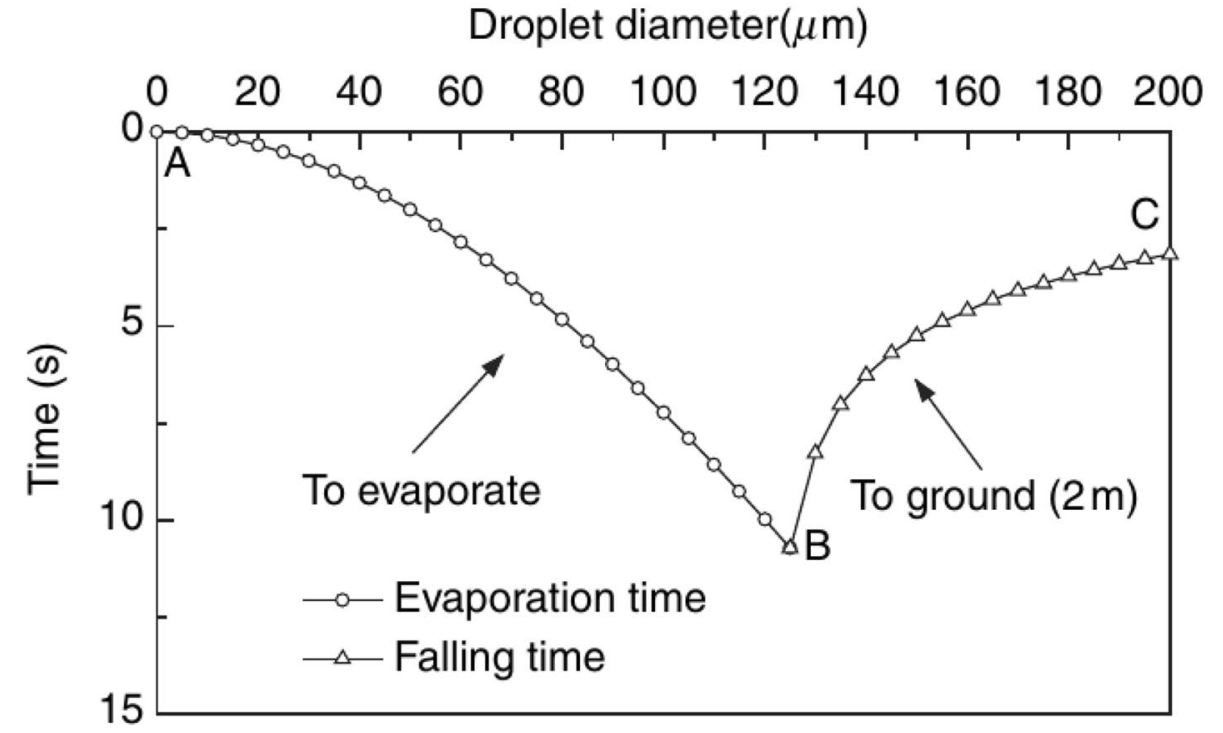


Figure 3 summarizes a list of common mitigation techniques, showing their effectiveness for the different transmission/infection mechanisms.

\subsection{Simple Mitigation Techniques for Large Droplets}

Large droplets follow a ballistic path. Their exit velocity is of the order of 2-14 m/s [17, 33-36, 100-102] for sneezing and coughing, and much lower for shouting and talking. This means that these droplets will reach the ground or any surface on their way to the ground within a distance of less than $2 \mathrm{~m}$. This is the basis of the so-called ' $2 \mathrm{~m}$ ' or ' $6 \mathrm{ft}$ ' rules/advisories issued by the Centers for Disease Control and Prevention (CDC) and the World Health Organization (WHO). The mitigation and elimination options for these droplets are the following:

- Distance: keeping sufficient distance is by far the most effective mitigation mechanism against infection via large droplets; hence the ' $2 \mathrm{~m}$ ' or ' $6 \mathrm{ft}$ ' distance guidance;

- Constant hand washing: large droplets will not only fall to the ground but stick to surfaces, leading to so-called 'fomite infection' when victims touch these surfaces and then their nose, eyes or mouth; constant hand washing is a simple way to mitigate this infection route;

- Face masks: any kind of face mask is extremely effective against large droplets; this applies both to emission (i.e. from an infected person to the environment) as well as inhalation (i.e. from the environment to a person);

- Plexiglass walls: plexiglass walls/shields offer a very effective barrier against large droplets; cashiers and receptionists regularly witness the considerable retaining power of these walls/shields when cleaning them at the end of a day;

- Face shields/visor masks: this is the 'personalized plexiglass shield'; as such, it is a very efficient barrier against large droplets; however, it needs careful handling: touching the outside to move/place/arrange the visor, something that is unavoidable and will occur periodically, means that hands will be in touch with an infected surface, something that can increase the danger of infection;

- Glasses: the occasional droplet hitting an eye is best avoided via glasses; as before, care is needed when touching/cleaning the glasses.

- Periodic $(2 \times$ day minimum) disinfectant swiping of all surfaces and plexiglass: in the same way that infection can be avoided by hand-washing, any surface that is closer than $2 \mathrm{~m}$ to any person in a room should be periodically swiped clean with disinfectant; as with hands, the more, the better; the recommendation is at a minimum $2 \times$ a day;

- Disinfectant cleaning of any object that changes hands: in courts, medical facilities, offices and many other buildings/venues objects change hands; these could be documents, papers, notes, etc.; these could become infection
Fig. 3 Common procedures to mitigate the spread of pathogens green: effective; yellow: somewhat effective; red: ineffective. (Colour figure online)

\begin{tabular}{|c|c|c|c|c|}
\hline $\begin{array}{l}\text { Procedure } \\
\text { Measure }\end{array}$ & $\begin{array}{l}\text { Large Droplets } \\
\text { (spitting) }\end{array}$ & $\begin{array}{l}\text { Small Droplets } \\
\text { (cigarette smoke) }\end{array}$ & $\begin{array}{l}\text { Person-Air- } \\
\text { Person }\end{array}$ & $\begin{array}{l}\text { Person-Surface- } \\
\text { Person }\end{array}$ \\
\hline \multicolumn{5}{|l|}{$2 \mathrm{~m} / 6 \mathrm{ft}$ Distance } \\
\hline \multicolumn{5}{|l|}{ Face Masks } \\
\hline \multicolumn{5}{|l|}{$\begin{array}{l}\text { Periodic Hand } \\
\text { Cleaning }\end{array}$} \\
\hline \multicolumn{5}{|l|}{$\begin{array}{l}\text { Plexiglass } \\
\text { Shields }\end{array}$} \\
\hline \multicolumn{5}{|l|}{$\begin{array}{l}\text { 1-Way Person } \\
\text { Traffic }\end{array}$} \\
\hline \multicolumn{5}{|l|}{$\begin{array}{l}\text { 2x Daily } \\
\text { Cleaning }\end{array}$} \\
\hline \multicolumn{5}{|l|}{$\begin{array}{l}\text { Nightly UV } \\
\text { Cleaning }\end{array}$} \\
\hline \multicolumn{5}{|l|}{$\begin{array}{l}\text { Maximize Fresh } \\
\text { Air in HVAC }\end{array}$} \\
\hline \multicolumn{5}{|l|}{$\begin{array}{l}\text { Hard UV Lamps } \\
\text { in HVAC Ducts }\end{array}$} \\
\hline \multicolumn{5}{|l|}{$\begin{array}{l}\text { HEPA Filters in } \\
\text { HVAC Ducts }\end{array}$} \\
\hline $\begin{array}{l}\text { Upper Room UV } \\
\text { Cleaning }\end{array}$ & & & & \\
\hline
\end{tabular}


sources; therefore, they should be cleaned with disinfectant before changing hands;

- 'Virus elimination' storage of other objects that change hands: if any object that changes hands is not immediately needed, it could be stored for $24-48 \mathrm{~h}$ in a dry storage room; the virus will die naturally in this timeframe.

\subsection{Simple Mitigation Techniques for Small Droplets}

Small droplets follow the movement of air [3-5]. As stated before, one should consider the movement of these small droplets like the movement of cigarette smoke. Any nonsmoker who had smokers among family or acquaintances can recall how difficult it is to mitigate the smell and contamination of cigarette smoke.

- Ventilation: the best way to avoid infection via small droplets is a constant supply of fresh air; this is not always simple, particularly for large buildings with many 'interior rooms';

- HVAC Filters: given that in modern, energy efficient HVAC systems less than $30 \%$ of the air is 'new, fresh air from outside', implying that the air recirculated 3-4 times, it is of paramount importance to have the best possible filters in the HVAC systems; filter rating follow the so-called MERV-scale. The higher the MERV-rating, the better the filter; the CDC currently mandates MERV-8, but recommends MERV-13;

- Avoidance of wakeflow: given that these droplets propagate with the air, one should make sure not to be placed in the 'exhale-path' or 'wake-path' of another person; the best way to conceptualize this is by considering any other person a smoker, and placing oneself 'away from the smoke'; a simple way of realizing this is making sure that there is an airflow across (from right to left or left to right) between two talking individuals;

- Face masks: any kind of face mask is also effective against small droplets; however, the effectivity is not as high as compared to large droplets [52,85]; in order to shield oneself from small droplets N95 (or higher) masks are required;

- Plexiglass walls: plexiglass walls/shields only offer an effective barrier against small droplets if they divert the airflow between the spatial sections; this is not always the case, and requires careful design and sizing; as before, think and visualize these small droplets as invisible cigarette smoke;

- Face shields/visor masks: are practically useless against cigarette smoke, and hence also against small droplets;

- Pedestrian traffic management: Many large buildings have a single area for entrance/exit. This will lead to pedestrian counterflows, which in turn will increase the turbulence of air, the level of mixing and the chances of viral transmission via small droplets. A simple way to reduce this risk is to enforce 'single lane' pedestrian traffic. Better yet, separate entrances and exits so that these pedestrian streams do not encounter each other or mix at all.

\subsection{Advanced Mitigation Techniques}

Besides the simpler mitigation mechanisms described above, a number of advanced technologies have emerged that can help mitigate viral propagation and infection.

- Ultra-violet $(U V)$ radiation for surfaces: UV radiation destroys links in the DNA and RNA chains of living organisms, and has been used extensively in the food industry and water treatment plants to kill pathogens $[6,45]$. UV radiation can be used to 'clean' rooms from viruses. For example, it is estimated that a radiation load of $I_{0}=1.5 \times 10^{-3} \mathrm{~J} / \mathrm{cm}^{2}$ inactivates roughly $95 \%$ of Covid-19 viral matter. However, as UV irradiation is harmful to humans, the 'cleaning' needs to happen when buildings are not in use ('off-hour cleaning') and under the supervision of trained personnel. As optical shadowing is common for complex interiors, care has to be taken to place the UV lamps properly and move them so that all surfaces are sufficiently irradiated. A possible drawback of UV radiation is the much faster destruction or 'aging' of surfaces, particularly wooden or plastic surfaces.

- Ultra-violet $(U V)$ radiation for air in HVAC ducts: UV radiation can also be used to 'clean' the air. This is accomplished by placing UV lamps in heating, ventilation and air conditioning (HVAC) ducts $[6,16]$. In this way, any air passing through these ducts is irradiated, and any pathogens present are killed. As the lamps are in the HVAC ducts, the harmful UV radiation is contained and does not reach the outside. A possible drawback of placing UV lamps in HVAC ducts is the heat generated by them-not a problem in winter but certainly in summer.

- Ultra-violet $(U V)$ ceiling radiation for air in rooms: UV radiation can also be used to 'clean' the air in those parts of the room where people will not be present. This can be accomplished by special UV lamps that are placed in such a way that they only irradiate the upper part of

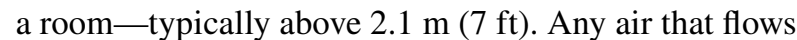
into/past those regions is continuously irradiated, as are pathogens present in it, eventually killing them. Some of the surfaces may reflect UV rays (visible light reflectivity differs from UV reflectivity, so this has to be measured), thus harming people in the rooms if not installed properly. The UV rays will also attack any surface composed of organic materials, changing their colour and in some cases weakening them. One should also bear in mind that 
if the rooms are properly ventilated, air will be exchanged at least 4-5 times an hour. Therefore, the particles will reside in the regions that are irradiated by UV for less than $15 \mathrm{~min}$, implying that a stong radiation is required to kill the pathogens 'in the first pass'.

- High efficiency particulate air (HEPA) filters: for decades, HVAC systems have been designed with energy efficiency as a primary goal. This has led to a minimization of fresh air supply, as well as very efficient mixing devices (so that occupants feel comfortable and rooms do not exhibit large variations in temperature). Both of these factors, i.e. minimization of fresh air and efficient mixing are highly conducive to spreading pathogens [67]. One of the ways to keep the fresh air supply at a low level and the air free of pathogens is the use of high efficiency particulate air (HEPA) filters. These will retain more than 99\% of the pathogens. HEPA filters have been installed in airplanes for many years. One of the disadvantages is the higher energy consumption: the air has to be pressed through tiny pores, requiring high pressures to achieve the desired flowrates.

\section{Modeling Pathogen Transmission and Infection}

Pathogen, or more generally, disease transmission models have a long history in both health sciences and applied mathematical modeling since the seminal work of Wells [112]. The probability of a person getting infected through the airborne route depends on

- That person's exposure to the pathogen/virus (the dose) and

- The probability of getting infected given that level of exposure [98].

The first term depends on a variety of factors such as pathogens shed by the infective person, exposure time, air circulation patterns, etc. [37, 98]. In aggregate models, which include the vast majority of models, the specific locations of susceptible persons are not explicitly considered. Rather, one makes assumptions so that these locations do not have to be specified. For example, the viral particles may be assumed to be uniformly distributed, in which case the specific locations of persons are not required. More sophisticated models can account for spatial heterogeneity by dividing the space into multiple zones with different distributions of viral particles in each zone [2]. This requires knowing only the exposure times of persons in each zone, and not their specific positions. In individualized models, which are relatively rare, the specific positions of persons are required. For example, Gupta et al. [37] consider specific seats in which passengers in a plane are seated and examine their exposure using computational fluid dynamics (CFD) simulations.

The second term-the relationship between exposure and infection probability-can be deterministic or probabilistic $[37,98]$, with the latter being much more common. In a deterministic model, a person is considered infected if that person inhales more viral particles than a limit, called that person's tolerance dose. In a probabilistic model, on the other hand, the infection probability depends on the extent of exposure. Models typically use variants of one of the following two approaches. In both approaches, infection probability is given by

$p=1-e^{-c \cdot e_{m}}$

where $c$ is a constant and $e_{m}$ the so-called exposure metric. In the Wells-Riley approach [112], the exposure is expressed in terms of an abstract 'quantum' of infection, whose relative values can be computed for different scenarios, and model parameters determined by fitting against empirical data. In a dose-response model, on the other hand, the exposure metric reflects the actual number of viral particles inhaled by the susceptible person, with detailed mechanisms for computing this value.

\subsection{Relationship to Previous Work}

Conventional models are aggregate, and do not consider the specific locations of individuals. Consequently, they cannot account for fine-scaled spatial heterogeneity. Individual models, such as [37], do consider positions of individuals. However, they consider situations where the positions of persons are fixed. This is inadequate for understanding risk associated when people move in a crowd. Namilae et al. [86] consider movement of people in a plane using pedestrian dynamics. However, that work does not account for movement of viral particles through the air. Instead, it considers infection risk based on contacts between persons in the crowd.

\section{Requirements for Modeling Pathogen Propagation, Transmission and Mitigation}

Taking into account all the information stated before, one can see that in order to arrive at advanced numerical models to compute with high fidelity pathogen propagation, transmission and mitigation the following capabilities are required: 
- Physical modeling of sneezing/coughing (exit velocities and temperature, number and distribution of particles, ...);

- Physical modeling of aerosol propagation (flows with particles in an environment with moving pedestrians, geometric fidelity of the built environment, HVAC boundary conditions, ...);

- Physical modeling of UV radiation and the accumulated dosage on particles (placement of UV lamps, shadowing, ...);

- Modeling of pedestrian motion (movement, proximity, ...);

- Monitoring of pathogens exhaled and inhaled.

These in turn will enable the generation of the four essential pieces of information required:

- The generation of pathogen (e.g. viral) loads;

- The movement (advection, diffusion) of pathogen loads;

- The location and movement of pedestrians exhaling pathogens;

- The location and movement of pedestrians inhaling pathogens.

In the sequel, we will consider each one of these in turn. One should state from the outset that all of these quantities can vary greatly, so that any kind of model will have to be run repeatedly in order to obtain proper statistics.

\section{Physical Modeling of Aerosol Propagation}

When solving the two-phase equations, the air, as a continuum, is best represented by a set of partial differential equations (the Navier-Stokes equations) that are numerically solved on a mesh. Thus, the gas characteristics are calculated at the mesh points within the flowfield. The droplets/ particles, which are relatively sparse in the flowfield, are modeled using a Lagrangian description, where individual particles (or groups of particles) are monitored and tracked in the flow, allowing for an exchange of mass, momentum and energy between the air and the particles.

\subsection{Equations Describing the Motion of the Air}

As seen from the experimental evidence, the velocities of air encountered during coughing and sneezing never exceed a Mach-number of $M a=0.1$. Therefore, the air may be assumed as a Newtonian, incompressible liquid, where buoyancy effects are modeled via the Boussinesq approximation. The equations describing the conservation of momentum, mass and energy for incompressible, Newtonian flows may be written as $\rho \mathbf{v}_{, t}+\rho \mathbf{v} \cdot \nabla \mathbf{v}+\nabla p=\nabla \cdot \mu \nabla \mathbf{v}+\rho \mathbf{g}+\beta \rho \mathbf{g}\left(T-T_{0}\right)+\mathbf{s}_{v}$

$\nabla \cdot \mathbf{v}=0$

$\rho c_{p} T_{, t}+\rho c_{p} \mathbf{v} \cdot \nabla T=\nabla \cdot k \nabla T+s_{e}$.

Here $\rho, \mathbf{v}, p, \mu, \mathbf{g}, \beta, T, T_{0}, c_{p}, k$ denote the density, velocity vector, pressure, viscosity, gravity vector, coefficient of thermal expansion, temperature, reference temperature, specific heat coefficient and conductivity respectively, and $\mathbf{s}_{v}, s_{e}$ momentum and energy source terms (e.g. due to particles or external forces/heat sources). For turbulent flows both the viscosity and the conductivity are obtained either from additional equations or directly via a large eddy simulation (LES) assumption through monotonicity induced LES (MILES) [9, 29, 30, 51].

The transport of pathogens in aerosols with very small particles may be modeled by particles or as a continuum (a justification for this assumption is given below after the description of particle motion). In the latter case, an advection-diffusion equation of the form:

$c_{, t}+\mathbf{v} \cdot \nabla c=\nabla \cdot k_{c} \nabla c+s_{c}$,

is required, where $c, k_{c}$ denote the concentration and diffusivity of the pathogen, and $s_{c}$ is the source (or sink) term (due to exhalation or inhalation). In addition, a series of additional 'diagnostics' equations may be required, such as: 'the age of air' $\tau$ (a good measure for ventilation efficiency):

$\tau_{, t}+\mathbf{v} \cdot \nabla \tau=1$,

and the accumulated radiation $(r)$ :

$r_{, t}+\mathbf{v} \cdot \nabla r=I$,

where $I$ denotes the local irradiation intensity.

\subsection{Equations Describing the Motion of Particles/ Droplets}

In order to describe the interaction of particles/droplets with the flow, the mass, forces and energy/work exchanged between the flowfield and the particles must be defined. As before, we denote for fluid (air) by $\rho, p, T, k, v_{i}, \mu$ and $c_{p}$ the density, pressure, temperature, conductivity, velocity in direction $x_{i}$, viscosity, and the specific heat at constant pressure. For the particles, we denote by $\rho_{p}, T_{p}, v_{p i}, d, c_{p p}$ and $Q$ the density, temperature, velocity in direction $x_{i}$, equivalent diameter, specific heat coefficient and heat transferred per unit volume. In what follows we will refer to droplet and particles collectively as particles.

Making the classical assumptions that the particles may be represented by an equivalent sphere of diameter $d$, the 
drag forces $\mathbf{D}$ acting on the particles will be due to the difference of fluid and particle velocity:

$\mathbf{D}=\frac{\pi d^{2}}{4} \cdot c_{d} \cdot \frac{1}{2} \rho\left|\mathbf{v}-\mathbf{v}_{p}\right|\left(\mathbf{v}-\mathbf{v}_{p}\right)$.

The drag coefficient $c_{d}$ is obtained empirically from the Reynolds-number $R e$ :

$\operatorname{Re}=\frac{\rho\left|\mathbf{v}-\mathbf{v}_{p}\right| d}{\mu}$

as (see, e.g. [95]):

$c_{d}=\max \left(0.1, \frac{24}{R e}\left(1+0.15 R e^{0.687}\right)\right)$

The lower bound of $c_{d}=0.1$ is required to obtain the proper limit for the Euler equations, when $R e \rightarrow \infty$. The heat transferred between the particles and the fluid is given by

$Q=\frac{\pi d^{2}}{4} \cdot\left[h_{f} \cdot\left(T-T_{p}\right)+\sigma^{*} \cdot\left(T^{4}-T_{p}^{4}\right)\right]$,

where $h_{f}$ is the film coefficient and $\sigma^{*}$ the radiation coefficient. For the class of problems considered here, the particle temperature and kinetic energy are such that the radiation coefficient $\sigma^{*}$ may be ignored. The film coefficient $h_{f}$ is obtained from the Nusselt number $\mathrm{Nu}$ :

$N u=2+0.459 \operatorname{Pr}^{0.333} \operatorname{Re}^{0.55}$,

where $\operatorname{Pr}$ is the Prandtl number of the gas

$\operatorname{Pr}=\frac{k}{\mu}$,

as

$h_{f}=\frac{N u \cdot k}{d}$.

Having established the forces and heat flux, the particle motion and temperature are obtained from Newton's law and the first law of thermodynamics. For the particle velocities, we have:

$\rho_{p} \frac{\pi d^{3}}{6} \cdot \frac{d \mathbf{v}_{p}}{d t}=\mathbf{D}+\rho_{p} \frac{\pi d^{3}}{6} \mathbf{g}$.

This implies that:

$\frac{d \mathbf{v}_{p}}{d t}=\frac{3 \rho}{4 \rho_{p} d} \cdot c_{d}\left|\mathbf{v}-\mathbf{v}_{p}\right|\left(\mathbf{v}-\mathbf{v}_{p}\right)+\mathbf{g}=\alpha_{v}\left|\mathbf{v}-\mathbf{v}_{p}\right|\left(\mathbf{v}-\mathbf{v}_{p}\right)+\mathbf{g}$,

where $\alpha_{v}=3 \rho c_{d} /\left(4 \rho_{p} d\right)$. The particle positions are obtained from: $\frac{d \mathbf{x}_{p}}{d t}=\mathbf{v}_{p}$

The temperature change in a particle is given by:

$\rho_{p} c_{p p} \frac{\pi d^{3}}{6} \cdot \frac{d T_{p}}{d t}=Q$

which may be expressed as:

$\frac{d T_{p}}{d t}=\frac{3 k}{2 c_{p p} \rho_{p} d^{2}} \cdot N u \cdot\left(T-T_{p}\right)=\alpha_{T}\left(T-T_{p}\right)$,

with $\alpha_{T}=3 k N u /\left(2 c_{p p} \rho_{p} d^{2}\right)$. Equations $(6.2 .9,6.2 .10$, 6.2.12) may be formulated as a system of ordinary differential equations (ODEs) of the form:

$\frac{d \mathbf{u}_{p}}{d t}=\mathbf{r}\left(\mathbf{u}_{p}, \mathbf{x}, \mathbf{u}_{f}\right)$,

where $\mathbf{u}_{p}, \mathbf{x}, \mathbf{u}_{f}$ denote the particle unknowns, the position of the particle and the fluid unknowns at the position of the particle.

In order to see whether a fully coupled particleflow simulation is required in the present context, consider the motion of a water particle with an initial velocity of $v_{i}=1 \mathrm{~m} / \mathrm{s}$ into quiescent air. Using the equations shown above, and the usual values of $\rho_{p}=1000 \mathrm{~kg} / \mathrm{m}^{3}, \rho_{\text {air }}=1.2 \mathrm{~kg} / \mathrm{m}^{3}, \mu_{\text {air }}=1.85 \times 10^{-5} \mathrm{~kg} / \mathrm{m} / \mathrm{s}$, one can obtain the distance and time to rest, where 'rest' in this case is assumed as $v_{r}=0.01 v_{i}$. These values have been tabulated in Table 2. One can see that for diameters below $O(0.1 \mathrm{~mm})$ the time and distance required for a particle to adjust to the velocity of the surrounding air is so low that for these aerosol particles one can neglect the air-particle interaction. Therefore, one can treat these aerosol particles via the transport equation (Eq. 6.1.4).

\subsection{Numerical Integration of the Motion of the Air}

The last six decades have seen a large number of schemes that may be used to solve numerically the incompressible Navier-Stokes equations given by Eqs. (6.1.1-6.1.3). In the present case, the following design criteria were implemented:

Table 2 Distance and time to rest for water particles in air with an initial velocity of $1 \mathrm{~m} / \mathrm{s}$

\begin{tabular}{lll}
\hline Diameter $(\mathrm{mm})$ & Distance to rest $(\mathrm{m})$ & Time to rest $(\mathrm{s})$ \\
\hline $1.00 \mathrm{E}-01$ & $2.27 \mathrm{E}-02$ & $1.20 \mathrm{E}-01$ \\
$1.00 \mathrm{E}-02$ & $1.34 \mathrm{E}-03$ & $2.79 \mathrm{E}-04$ \\
$1.00 \mathrm{E}-03$ & $1.40 \mathrm{E}-05$ & $2.94 \mathrm{E}-06$ \\
\hline
\end{tabular}


- Spatial discretization using unstructured grids (in order to allow for arbitrary geometries and adaptive refinement);

- Spatial approximation of unknowns with simple linear finite elements (in order to have a simple input/output and code structure);

- Edge-based data structures (for reduced access to memory and indirect addressing);

- Temporal approximation using implicit integration of viscous terms and pressure (the interesting scales are the ones associated with advection);

- Temporal approximation using explicit, high-order integration of advective terms;

- Low-storage, iterative solvers for the resulting systems of equations (in order to solve large 3-D problems); and

- Steady results that are independent from the timestep chosen (in order to have confidence in convergence studies).

The resulting discretization in time is given by the following projection scheme [72, 73, 82]:

- Advective-Diffusive Prediction: $\mathbf{v}^{n}, p^{n} \rightarrow \mathbf{v}^{*}$

$$
\begin{aligned}
& \mathbf{s}^{\prime}=-\nabla p^{n}+\rho \mathbf{g}+\beta \rho \mathbf{g}\left(T^{n}-T_{0}\right)+\mathbf{s}_{v}, \\
& \mathbf{v}^{i}=\mathbf{v}^{n}+\alpha^{i} \gamma \Delta t\left(-\mathbf{v}^{i-1} \cdot \nabla \mathbf{v}^{i-1}+\nabla \cdot \mu \nabla \mathbf{v}^{i-1}+\mathbf{s}^{\prime}\right) ; i=\begin{array}{l}
1, k-1 ; \\
6.3 .2)
\end{array} \\
& {\left[\frac{1}{\Delta t}-\theta \nabla \cdot \mu \nabla\right]\left(\mathbf{v}^{k}-\mathbf{v}^{n}\right)+\mathbf{v}^{k-1} \cdot \nabla \mathbf{v}^{k-1}=\nabla \cdot \mu \nabla \mathbf{v}^{k-1}+\mathbf{s}^{\prime} .}
\end{aligned}
$$

- Pressure Correction: $p^{n} \rightarrow p^{n+1}$

$$
\begin{aligned}
& \nabla \cdot \mathbf{v}^{n+1}=0 ; \\
& \frac{\mathbf{v}^{n+1}-\mathbf{v}^{*}}{\Delta t}+\nabla\left(p^{n+1}-p^{n}\right)=0 ;
\end{aligned}
$$

which results in

$$
\nabla^{2}\left(p^{n+1}-p^{n}\right)=\frac{\nabla \cdot \mathbf{v}^{*}}{\Delta t}
$$

- Velocity Correction: $\mathbf{v}^{*} \rightarrow \mathbf{v}^{n+1}$

$$
\mathbf{v}^{n+1}=\mathbf{v}^{*}-\Delta t \nabla\left(p^{n+1}-p^{n}\right) .
$$

$\theta$ denotes the implicitness-factor for the viscous terms $(\theta=1: 1$ st order, fully implicit, $\theta=0.5: 2$ nd order, Crank-Nicholson). $\alpha^{i}$ are the standard low-storage Runge-Kutta coefficients $\alpha^{i}=1 /(k+1-i)$. The $k-1$ stages of Eq. (6.3.2) may be seen as a predictor (or replacement) of $\mathbf{v}^{n}$ by $\mathbf{v}^{k-1}$. The original right-hand side has not been modified, so that at steady-state $\mathbf{v}^{n}=\mathbf{v}^{k-1}$, preserving the requirement that the steady-state be independent of the timestep $\Delta t$. The factor $\gamma$ denotes the local ratio of the stability limit for explicit timestepping for the viscous terms versus the timestep chosen. Given that the advective and viscous timestep limits are proportional to:

$\Delta t_{a} \approx \frac{h}{|\mathbf{v}|} ; \Delta t_{v} \approx \frac{\rho h^{2}}{\mu}$,

we immediately obtain

$\gamma=\frac{\Delta t_{v}}{\Delta t_{a}} \approx \frac{\rho|\mathbf{v}| h}{\mu} \approx R e_{h}$,

or, in its final form:

$\gamma=\min \left(1, \operatorname{Re}_{h}\right)$.

In regions away from boundary layers, this factor is $O(1)$, implying that a high-order Runge-Kutta scheme is recovered. Conversely, for regions where $R e_{h}=O(0)$, the scheme reverts back to the usual 1-stage Crank-Nicholson scheme. Besides higher accuracy, an important benefit of explicit multistage advection schemes is the larger timestep one can employ. The increase in allowable timestep is roughly proportional to the number of stages used (and has been exploited extensively for compressible flow simulations [58]). Given that for an incompressible solver of the projection type given by Eqs. (6.3.1-6.3.7) most of the CPU time is spent solving the pressure-Poisson system (Eq. 6.3.6), the speedup achieved is also roughly proportional to the number of stages used.

At steady state, $\mathbf{v}^{*}=\mathbf{v}^{n}=\mathbf{v}^{n+1}$ and the residuals of the pressure correction vanish, implying that the result does not depend on the timestep $\Delta t$.

The spatial discretization of these equations is carried out via linear finite elements. The resulting matrix system is re-written as an edge-based solver, allowing the use of consistent numerical fluxes to stabilize the advection and divergence operators [73].

The energy (temperature) equation (Eq. 6.3.3) is integrated in a manner similar to the advective-diffusive prediction (Eq. 6.3.2), i.e. with an explicit, high order Runge-Kutta scheme for the advective parts and an implicit, 2nd order Crank-Nicholson scheme for the conductivity.

\subsection{Numerical Integration of the Motion of Particles/Droplets}

The equations describing the position, velocity and temperature of a particle (Eqs. 6.2.9, 6.2.10, 6.2.12) may be formulated as a system of nonlinear ordinary differential equations of the form: 
$\frac{d \mathbf{u}_{p}}{d t}=\mathbf{r}\left(\mathbf{u}_{p}, \mathbf{x}, \mathbf{u}_{f}\right)$.

They can be integrated numerically in a variety of ways. Due to its speed, low memory requirements and simplicity, we have chosen the following k-step low-storage Runge-Kutta procedure to integrate them:

$\mathbf{u}_{p}^{n+i}=\mathbf{u}_{p}^{n}+\alpha^{i} \Delta t \cdot \mathbf{r}\left(\mathbf{u}_{p}^{n+i-1}, \mathbf{x}^{n+i-1}, \mathbf{u}_{f}^{n+i-1}\right), i=1, k$.

For linear ODEs the choice

$\alpha^{i}=\frac{1}{k+1-i}, i=1, k$

leads to a scheme that is $k$ th order accurate in time. Note that in each step the location of the particle with respect to the fluid mesh needs to be updated in order to obtain the proper values for the fluid unknowns. The default number of stages used is $k=4$. This would seem unnecessarily high, given that the flow solver is of second-order accuracy, and that the particles are integrated separately from the flow solver before the next (flow) timestep, i.e. in a staggered manner. However, it was found that the 4-stage particle integration preserves very well the motion in vortical structures and leads to less 'wall sliding' close to the boundaries of the domain [77]. The stability/accuracy of the particle integrator should not be a problem as the particle motion will always be slower than the maximum wave speed of the fluid (fluid velocity).

The transfer of forces and heat flux between the fluid and the particles must be accomplished in a conservative way, i.e. whatever is added to the fluid must be subtracted from the particles and vice-versa. The finite element discretization of the fluid equations will lead to a system of ODE's of the form:

$\mathbf{M} \Delta \mathbf{u}=\mathbf{r}$,

where $\mathbf{M}, \Delta \mathbf{u}$ and $\mathbf{r}$ denote, respectively, the consistent mass matrix, increment of the unknowns vector and right-hand side vector. Given the 'host element' of each particle, i.e. the fluid mesh element that contains the particle, the forces and heat transferred to $\mathbf{r}$ are added as follows:

$\mathbf{r}_{D}^{i}=\sum_{\text {elsurr } i} N^{i}\left(\mathbf{x}_{p}\right) \mathbf{D}_{p}$

Here $N^{i}\left(\mathbf{x}_{p}\right)$ denotes the shape-function values of the host element for the point coordinates $\mathbf{x}_{p}$, and the sum extends over all elements that surround node $i$. As the sum of all shape-function values is unity at every point:

$\sum N^{i}(\mathbf{x})=1 \forall \mathbf{x}$

this procedure is strictly conservative.
From Eqs. $(6.2 .9,6.2 .10,6.2 .12)$ and their equivalent numerical integration via Eq. (6.4.2), the change in momentum and energy for one particle is given by:

$\mathbf{f}_{p}=\rho_{p} \frac{\pi d^{3}}{6} \frac{\left(\mathbf{v}_{p}^{n+1}-\mathbf{v}_{p}^{n}\right)}{\Delta t}$

$q_{p}=\rho_{p} c_{p p} \frac{\pi d^{3}}{6} \frac{\left(T_{p}^{n+1}-T_{p}^{n}\right)}{\Delta t}$.

These quantities are multiplied by the number of particles in a packet in order to obtain the final values transmitted to the fluid. Before going on, we summarize the basic steps required in order to update the particles one timestep:

- Initialize Fluid Source-Terms: $\mathbf{r}=0$

- DO: For Each Particle:

- DO: For Each Runge-Kutta Stage:

- Find Host Element of Particle: IELEM, $N^{i}(\mathbf{x})$

- Obtain Fluid Variables Required

- Update Particle: Velocities, Position, Temperature,

$-\quad$ ENDDO

- Transfer Loads to Element Nodes

- ENDDO

\subsubsection{Particle Parcels}

For a large number of very small particles, it becomes impossible to carry every individual particle in a simulation. The solution is to:

(a) Agglomerate the particles into so-called packets of $N_{p}$ particles;

(b) Integrate the governing equations for one individual particle; and

(c) Transfer back to the fluid $N_{p}$ times the effect of one particle.

Beyond a reasonable number of particles per element (typically $>8$ ), this procedure produces accurate results without any deterioration in physical fidelity [77].

\subsubsection{Other Particle Numerics}

In order to achieve a robust particle integrator, a number of additional precautions and algorithms need to be implemented. The most important of these are:

- Agglomeration/Subdivision of Particle Parcels: As the fluid mesh may be adaptively refined and coarsened in time, or the particle traverses elements of different sizes, 
it may be important to adapt the parcel concentrations as well. This is necessary to ensure that there is sufficient parcel representation in each element and yet, that there are not too many parcels as to constitute an inefficient use of CPU and memory.

- Limiting During Particle Updates: As the particles are integrated independently from the flow solver, it is not difficult to envision situations where for the extreme cases of very light or very heavy particles physically meaningless or unstable results may be obtained. In order to prevent this, the changes in particle velocities and temperatures are limited in order not to exceed the differences in velocities and temperature between the particles and the fluid [77].

- Particle Contact/Merging: In some situations, particles may collide or merge in a certain region of space.

- Particle Tracking: A common feature of all particlegrid applications is that the particles do not move far between timesteps. This makes physical sense: if a particle jumped ten gridpoints during one timestep, it would have no chance to exchange information with the points along the way, leading to serious errors. Therefore, the assumption that the new host elements of the particles are in the vicinity of the current ones is a valid one. For this reason, the most efficient way to search for the new host elements is via the vectorized neighbour-to-neighbour algorithm described in $[73,75]$.

\subsection{Immersed Body Techniques}

The information required from CCD codes consists of the pedestrians in the flowfield, i.e. their position, velocity, temperature, as well inhalation and exhalation. As the CCD codes describe the pedestrians as points, circles or ellipses, a way has to be found to transform this data into 3-D objects. Two possibilities have been pursued here:

(a) Transform each pedestrian into a set of (overlapping) spheres that approximate the body with maximum fidelity with the minimum amount of spheres;

(b) Transform each pedestrian into a set of tetrahedra that approximate the body with maximum fidelity with the minimum amount of tetrahedra.

The reason for choosing spheres or tetrahedra is that one can perform the required interpolation/information transfer much faster than with other methods.

In order to 'impose' on the flow the presence of a pedestrian the immersed boundary methodology is used. The key idea is to prescribe at every CFD point covered by a pedestrian the velocity and temperature of the pedestrian. For the CFD code, this translates into an extra set of boundary conditions that vary in time and space as the pedestrians move.
This is by now a mature technology (see, e.g. chapter 18 in [73] and the references cited therein). Fast search techniques as well as extensions to higher order boundary conditions may be found in $[73,76]$. Nevertheless, as the pedestrians potentially change location at every timestep, the search for and the imposition of new boundary conditions can add a considerable amount of CPU as compared to 'flow-only' runs.

\section{Physical Modeling of UV Radiation}

UV radiation destroys links in the DNA and RNA chains of living organisms, has been used extensively in the food industry and water treatment plants to kill pathogens [45], and is one of the mitigation procedures recommended by the CDC [32]. The radiation is emitted from UV lamps, and pathogens are considered inactive after receiving a sufficient amount of radiation, the so-called 'kill dosage'. These lamps have tables of radiation intensity that have been measured in an accredited laboratory setting. They consist of values of radiation intensity versus angles, and need to be interpolated accordingly. The radiation intensity is measured at a given distance from the lamp/source, and it is assumed that the radiation decays with the square of the distance. In order to obtain the accumulated irradiation of a particle in air or a parcel of fluid, the radiation intensity at every point in space is needed. Air acts as a transparent gas for UV radiation, implying that absorbtion of radiation in air is insignificant. Therefore, only the distance and relative angle position to each UV lamp is required. Given that due to other objects shadowing effects can occur, this implies the need for a fast algorithm to start at an arbitrary point in space and trace back to the lamp. For the linear (tetrahedral) elements employed here, this can be easily accomplished with the raytracing technique proposed in [26]. Let $\mathbf{x}_{\text {beg }}$ be the start point of the ray (either a point or Gauss quadrature point), and $\mathbf{x}_{\text {end }}$ be the end point of the ray (typically the lamp). Then the ray velocity vector is given by

$\mathbf{v}_{c}=c_{l} \frac{\mathbf{x}_{\text {end }}-\mathbf{x}_{\text {beg }}}{\left|\mathbf{x}_{\text {end }}-\mathbf{x}_{\text {beg }}\right|}$,

where $c_{l}$ denotes the speed of light.

With the notation of Fig. 4, any given point $\mathbf{x}_{p}$ can be expressed in terms of the non-dimensional coordinates $\xi, \eta, \zeta$ as:

$\mathbf{x}_{p}=\mathbf{x}_{A}+\left(\mathbf{x}_{B}-\mathbf{x}_{A}\right) \xi+\left(\mathbf{x}_{C}-\mathbf{x}_{A}\right) \eta+\left(\mathbf{x}_{D}-\mathbf{x}_{A}\right) \zeta=\mathbf{x}_{A}+\mathbf{G} \boldsymbol{\xi}$,

implying 


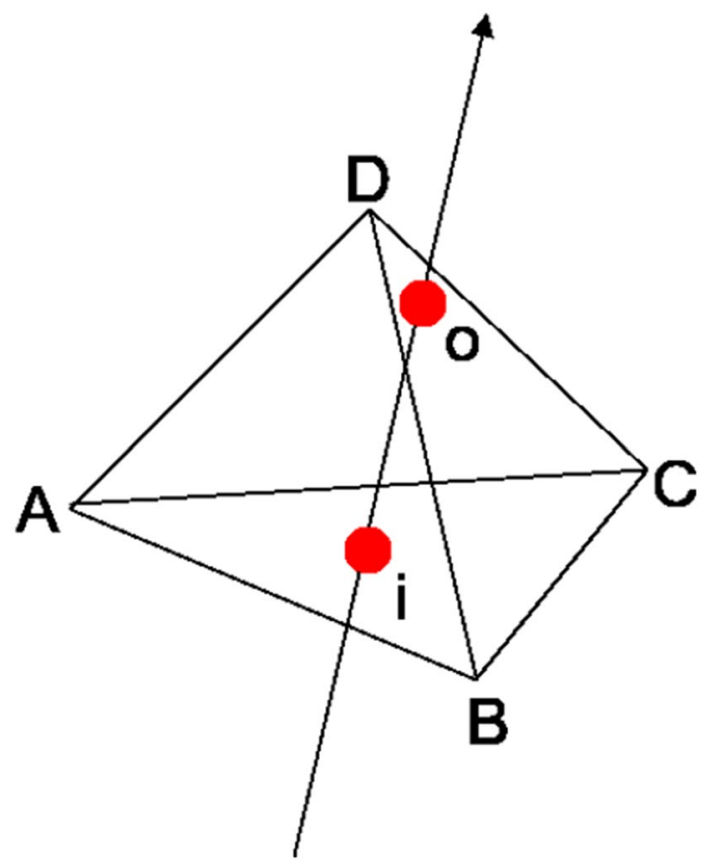

Fig. 4 Raytracing through a mesh of tetrahedra

$\boldsymbol{\xi}=\mathbf{G}^{-1}\left(\mathbf{x}_{p}-\mathbf{x}_{A}\right)$.

If one considers a given input or entry location $\mathbf{x}_{i}$ with nondimensional coordinates $\xi_{i}$ (see Fig. 4 ), the output or exit location will be given by:

$\xi_{o}=\xi_{i}+\mathbf{G}^{-1}\left(\mathbf{x}_{o}-\mathbf{x}_{i}\right)=\xi_{i}+\mathbf{G}^{-1} \mathbf{v}_{c} \Delta t=\xi_{i}+\alpha \Delta t$.
At any of the output or exit faces, the value of any of the shape functions $\xi, \eta, \zeta, 1-\xi-\eta-\zeta$ will be 0 . Setting each of these equal 0 will result in different timesteps $\Delta t$. A test is therefore conducted to obtain the smallest non-zero timestep. This yields the proper values for $\mathbf{x}_{o}$, as well as the face and the associated neighbour element to continue the search. If a boundary face is encountered (i.e. no neighbour element is found), shadowing is assumed. While this procedure is of complexity $O\left(N_{p} \cdot n_{r}\right)$, where $N_{p}$ denotes the number of points in the mesh and $n_{r}$ the average number of elements traversed from ray origin to lamp, it is very fast and in most cases only needs to be carried out at the beginning of a run (hard UV radiation should not be used in regions where humans walk). Figure 5 shows a hospital room with a soft UV lamp in the ceiling (i.e. a radiation that does not penetrate the human skin). In this case, a constant value for the radiation is stored in the element, which is obtained by integrating the radiation seen by 5 Gauss quadrature points. Note the shadowing effects.

\section{Modeling of Pedestrian Motion}

The modeling of pedestrian motion has been the focus of research and development for more than two decades. If one is only interested in average quantities (average density, velocity), continuum models [28, 50, 89] are an option. For problems requiring more realism, approaches that model each individual are required [105]. Among these, discrete space models (such as cellular automata $[7,8,19,24,62,64,66,93,96$, 103]), force-based models (such as the social force model [41, $42,65,74,90])$ and agent-based techniques $[20,38,39,61$,
Fig. 5 Surface radiation from ceiling UV lamp

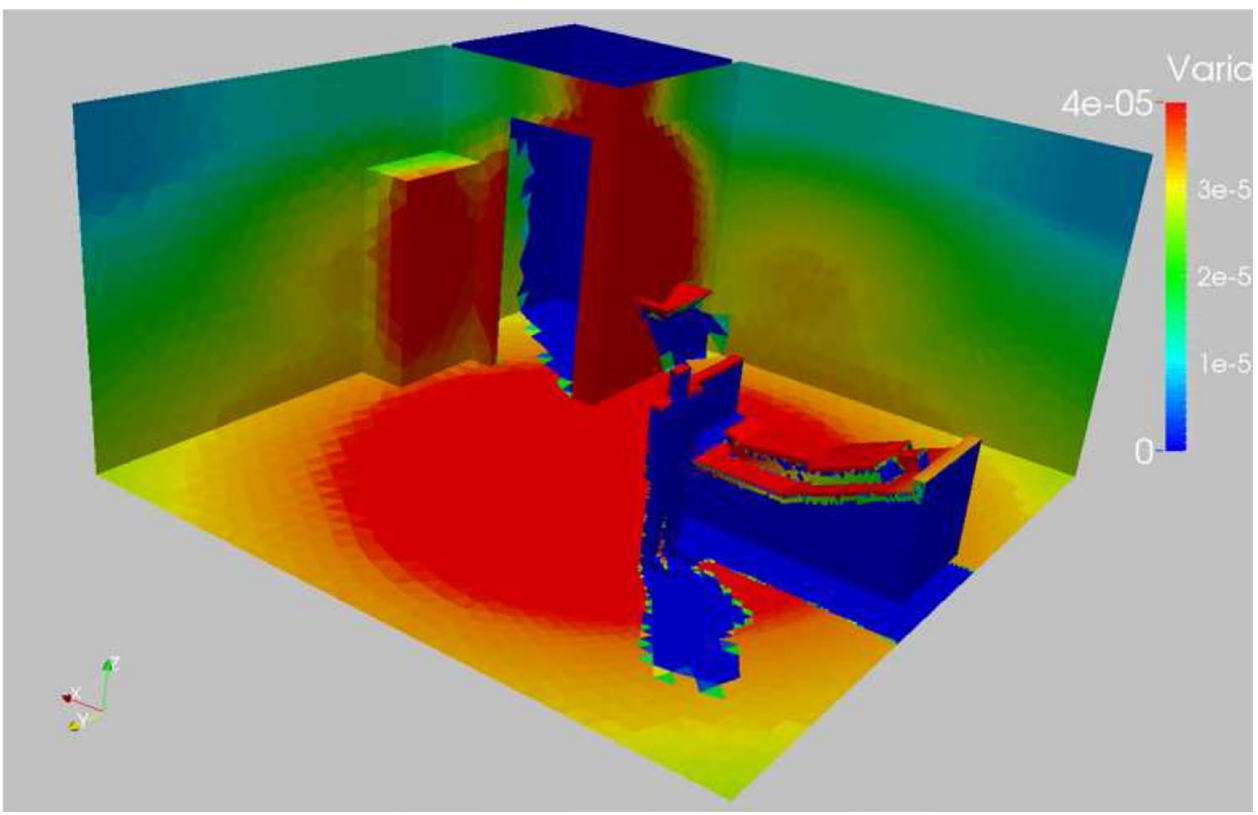


$87,88,97,106,108$ ] have been explored extensively. Together with insights from psychology and neuroscience (e.g. [106, 110]) it has become clear that any pedestrian motion algorithm that attempts to model reality should be able to mirror the following empirically known facts and behaviours:

- Newton's laws of motion apply to humans as well: from one instant to another, we can only move within certain bounds of acceleration, velocity and space;

- Contact between individuals occurs for high densities; these forces have to be taken into account;

- Humans have a mental map and plan on how they desire to move globally (e.g. first go here, then there, etc.);

- Human motion is therefore governed by strategic (long term, long distance), tactical (medium term, medium distance) and operational (immediate) decisions;

- In even moderately crowded situations $\left(O\left(1 \mathrm{p} / \mathrm{m}^{2}\right)\right)$, humans have a visual horizon of $O(2.5-5.0 \mathrm{~m})$, and a perception range of 120 degrees; thus, the influence of other humans beyond these thresholds is minimal;

- Humans have a 'personal comfort zone'; it is dependent on culture and varies from individual to individual, but it cannot be ignored;

- Humans walk comfortably at roughly 2 paces per second (frequency: $v=2 \mathrm{~Hz}$ ); they are able to change the frequency for short periods of time, but will return to $2 \mathrm{~Hz}$ whenever possible.

We remark that many of the important and groundbreaking work cited previously took place within the gaming/visualization community, where the emphasis is on 'looking right'. Here, the aim is to answer civil engineering or safety questions such as maximum capacity, egress times under emergency, or comfort. Therefore, comparisons with experiments and actual data are seen as essential [54, 55, 74].

\subsection{The PEDFLOW Model}

The PEDFLOW model $[74,78]$ incorporates these requirements as follows: individuals move according to Newton's laws of motion; they follow (via will forces) 'global movement targets'; at the local movement level, the motion also considers the presence of other individuals or obstacles via avoidance forces (also a type of will force) and, if applicable, contact forces. Newton's laws:

$m \frac{d \mathbf{v}}{d t}=\mathbf{f}, \frac{d \mathbf{x}}{d t}=\mathbf{v}$,

where $m, \mathbf{v}, \mathbf{x}, \mathbf{f}, t$ denote, respectively, mass, velocity, position, force and time, are integrated in time using a 2nd order explicit timestepping technique. The main modeling effort is centered on $\mathbf{f}$. In the present case the forces are separated into internal (or will) forces [I would like to move here or there] and external forces [I collided with another pedestrian or an obstacle]. For the sake of completeness, we briefly review the main forces used. For more information, as well as verification and validation studies, see [56-57, 74, 115].

\subsubsection{Will Force}

Given a desired velocity $\mathbf{v}_{d}$ and the current velocity $\mathbf{v}$, this force will be of the form

$\mathbf{f}_{\text {will }}=g_{w}\left(\mathbf{v}_{d}-\mathbf{v}\right)$.

The modelling aspect is included in the function $g_{w}$, which, in the non-linear case, may itself be a function of $\mathbf{v}_{d}-\mathbf{v}$. Suppose $g_{w}$ is constant, and that only the will force is acting. Furthermore, consider a pedestrian at rest. In this case, we have:

$m \frac{d \mathbf{v}}{d t}=g_{w}\left(\mathbf{v}_{d}-\mathbf{v}\right), \mathbf{v}(0)=0$,

which implies:

$\mathbf{v}=\mathbf{v}_{d}\left(1-e^{-\alpha t}\right), \alpha=\frac{g_{w}}{m}=\frac{1}{t_{r}}$,

and

$\frac{d \mathbf{v}}{d t}(t=0)=\alpha \mathbf{v}_{d}=\frac{\mathbf{v}_{d}}{t_{r}}$.

One can see that the crucial parameter here is the 'relaxation time' $t_{r}$ which governs the initial acceleration and 'time to desired velocity'. Typical values are $v_{d}=1.35 \mathrm{~m} / \mathrm{s}$ and $t_{r}=O(0.5 \mathrm{~s})$. The 'relaxation time' $t_{r}$ is clearly dependent on the fitness of the individual, the current state of stress, desire to reach a goal, climate, signals, noise, etc. Slim, strong individuals will have low values for $t_{r}$, whereas obese or weak individuals will have high values for $t_{r}$. Furthermore, dividing by the mass of the individual allows all other forces (obstacle and pedestrian collision avoidance, contact, etc.) to be scaled by the 'relaxation time' as well, simplifying the modeling effort considerably.

The direction of the desired velocity

$\mathbf{s}=\frac{\mathbf{v}_{d}}{\left|\mathbf{v}_{d}\right|}$

will depend on the type of pedestrian and the cases considered. A single individual will have as its goal a desired position $\mathbf{x}_{d}\left(t_{d}\right)$ that he would like to reach at a certain time $t_{d}$. If there are no time constraints, $t_{d}$ is simply set to a large number. Given the current position $\mathbf{x}$, the direction of the velocity is given by 
$\mathbf{s}=\frac{\mathbf{x}_{d}\left(t_{d}\right)-\mathbf{x}}{\left|\mathbf{x}_{d}\left(t_{d}\right)-\mathbf{x}\right|}$,

where $\mathbf{x}_{d}\left(t_{d}\right)$ denotes the desired position (location, goal) of the pedestrian at the desired time of arrival $t_{d}$. For members of groups, the goal is always to stay close to the leader. Thus, $\mathbf{x}_{g}\left(t_{g}\right)$ becomes the position of the leader. In the case of an evacuation simulation, the direction is given by the gradient of the perceived time to exit $\tau_{e}$ to the closest perceived exit:

$\mathbf{s}=\frac{\nabla \tau_{e}}{\left|\nabla \tau_{e}\right|}$

The magnitude of the desired velocity $\left|\mathbf{v}_{d}\right|$ depends on the fitness of the individual, and the motivation/urgency to reach a certain place at a certain time. Pedestrians typically stroll leisurely at $0.6-0.8 \mathrm{~m} / \mathrm{s}$, walk at $0.8-1.0 \mathrm{~m} / \mathrm{s}$, jog at $1.0-3.0 \mathrm{~m} / \mathrm{s}$, and run at $3.0-10.0 \mathrm{~m} / \mathrm{s}$.

\subsubsection{Pedestrian Avoidance Forces}

The desire to avoid collisions with other individuals is modeled by first checking if a collision will occur. If so, forces are applied in the direction normal and tangential to the intended motion. The forces are of the form:

$f_{i}=f_{\text {max }} /\left(1+\rho^{p}\right) ; \rho=\left|\mathbf{x}_{i}-\mathbf{x}_{j}\right| / r_{i}$,

where $x_{i}, x_{j}$ denote the positions of individuals $i, j, r_{i}$ the radius of individual $i$, and $f_{\text {max }}=O(4) f_{\text {max }}$ (will). Note that the forces weaken with increasing non-dimensional distance $\rho$. For years we have used $p=2$, but, obviously, this can (and probably will) be a matter of debate and speculation (perhaps a future experimental campaign will settle this issue). In the far range, the forces are mainly orthogonal to the direction of intended motion: humans tend to move slightly sideways without decelerating. In the close range, the forces are also in the direction of intended motion, in order to model the slowdown required to avoid a collision.

\subsubsection{Wall Avoidance Forces}

Any pedestrian modeling software requires a way to input geographical information such as walls, entrances, stairs, escalators, etc. In the present case, this is accomplished via a triangulation (the so-called background mesh). A distance to walls map (i.e. a function $d_{w}(x)$ is constructed using fast marching techniques on unstructured grids), and this allows to define a wall avoidance force as follows:

$$
\mathbf{f}=-f_{\max } \frac{1}{1+\left(\frac{d_{w}}{r}\right)^{p}} \cdot \nabla d_{w}, p=2
$$

Note that $\left|\nabla d_{w}\right|=1$. The default for the maximum wall avoidance force is $f_{\max }=O(8) f_{\max }$ (will). The desire to be far/close to a wall also depends on cultural background.

\subsubsection{Contact Forces}

When contact occurs, the forces can increase markedly. Unlike will forces, contact forces are symmetric. Defining

$\rho_{i j}=\left|\mathbf{x}_{i}-\mathbf{x}_{j}\right| /\left(r_{i}+r_{j}\right)$,

these forces are modeled as follows:

$\rho_{i j}<1: f=-\left[f_{\max } /\left(1+\rho_{i j}^{p}\right)\right] ; p=2$

$\rho_{i j}>1: f=-\left[2 f_{\text {max }} /\left(1+\rho_{i j}^{p}\right)\right] ; p=2$

and $f_{\text {max }}=O(8) f_{\text {max }}($ will $)$.

\subsubsection{Motion Inhibition}

A key requirement for humans to move is the ability to put one foot in front of the other. This requires space. Given the comfortable walking frequency of $v=2 \mathrm{~Hz}$, one is able to limit the comfortable walking velocity by computing the distance to nearest neighbors and seeing which one of these is the most 'inhibiting'.

\subsubsection{Psychological Factors}

The present pedestrian motion model also incorporates a number of psychological factors that, among the many tried over the years, have emerged as important for realistic simulations. Among these, we mention:

- Determination/Pushiness: it is an everyday experience that in crowds, some people exhibit a more polite behavior than others. This is modeled in PEDFLOW by reducing the collision avoidance forces of more determined or 'pushier' individuals. Defining a determination or pushiness parameter $p$, the avoidance forces are reduced by $(1-p)$.

- Comfort zone: in some cultures (northern Europeans are a good example) pedestrians want to remain at some minimum distance from contacting others. This comfort zone is an input parameter in PEDFLOW, and is added to the radii of the pedestrians when computing collisions avoidance and pre-contact forces.

- Right/Left Avoidance and Overtaking: in many western countries pedestrians tend to avoid incoming pedestrians by stepping towards their right, and overtake others on the left. However, this is not the norm everywhere, and one has to account for it. 


\subsection{Numerical Integration of the Motion of Pedestrians}

The equations describing the position and velocity of a pedestrian may be formulated as a system of nonlinear Ordinary Differential Equations of the form:

$\frac{d \mathbf{u}_{p}}{d t}=\mathbf{r}\left(\mathbf{u}_{p}, \mathbf{x}, \mathbf{u}_{f}\right)$.

These ODEs are integrated with explicit Runge-Kutta schemes, typically of order 2 .

The geographic information required, such as terrain data (inclination, soil/water, escalators, obstacles, etc.), climate data (temperature, humidity, sun/rain, visibility), signs, the location and accessibility of guidance personnel, as well as doors, entrances and emergency exits is stored in a so-called background grid consisting of triangular elements. This background grid is used to define the geometry of the problem. At every instance, a pedestrian will be located in one of the elements of the background grid. Given this 'host element' the geographic data, stored at the nodes of the background grid, is interpolated linearly to the pedestrian. The closest distance to a wall $\delta_{w}$ or exit(s) for any given point of the background grid evaluated via a fast $(O(N \ln (N)))$ nearest neighbour/heap list technique [73, 74]. For cases with visual or smoke impediments, the closest distance to exit(s) is recomputed every few seconds of simulation time.

\subsection{Linkage to CFD Codes}

The information required from CFD codes such as FEFLO consists of the spatial distribution of temperature, smoke, other toxic or movement impairing substances in space, as well as pathogen distribution. This information is interpolated to the (topologically 2-D) background mesh at every timestep in order to calculate properly the visibility/reachability of exits, routing possibilities, smoke, toxic substance or pathogen inhalation, and any other flowfield variable required by the pedestrians. As the tetrahedral grid used for the CFD code and the triangular background grid of the CCD code do not change in time, the interpolation coefficients need to be computed just once at the beginning of the coupled run. While the transfer of information from CFD to $\mathrm{CCD}$ is voluminous, it is very fast, adding an insignificant amount to the total run-times.

\section{Coupling Methodology}

The coupling methodology used is shown in Fig. 6. The CFD code computes the flowfield, providing such information as temperature, smoke, toxic substance and pathogen concentration, and any other flow quantity that may affect the movement of pedestrians. These variables are then interpolated to the position where the pedestrians are, and are used with all other pertinent information (e.g. will-forces, targets, exits, signs, etc.) to update the position, velocity, inhalation of smoke, toxic substances or pathogens, state of exhaustion or intoxication, and any other pertinent quantity that is evaluated for the pedestrians. The position, velocity and temperature of the pedestrians, together with information such as sneezing or exhaling air, is then transferred to the CFD code and used to modify and update the boundary conditions of the flowfield, particles and pathogen concentrations in the regions where pedestrians are present.

Of the many possible coupling options (see e.g. [11, $15,94]$ ), we have implemented the simplest one: loose coupling with sequential timestepping [71, 83]. This is justified, as the timesteps of both the flow and pedestrian

Fig. 6 Coupling CFD and CCD codes

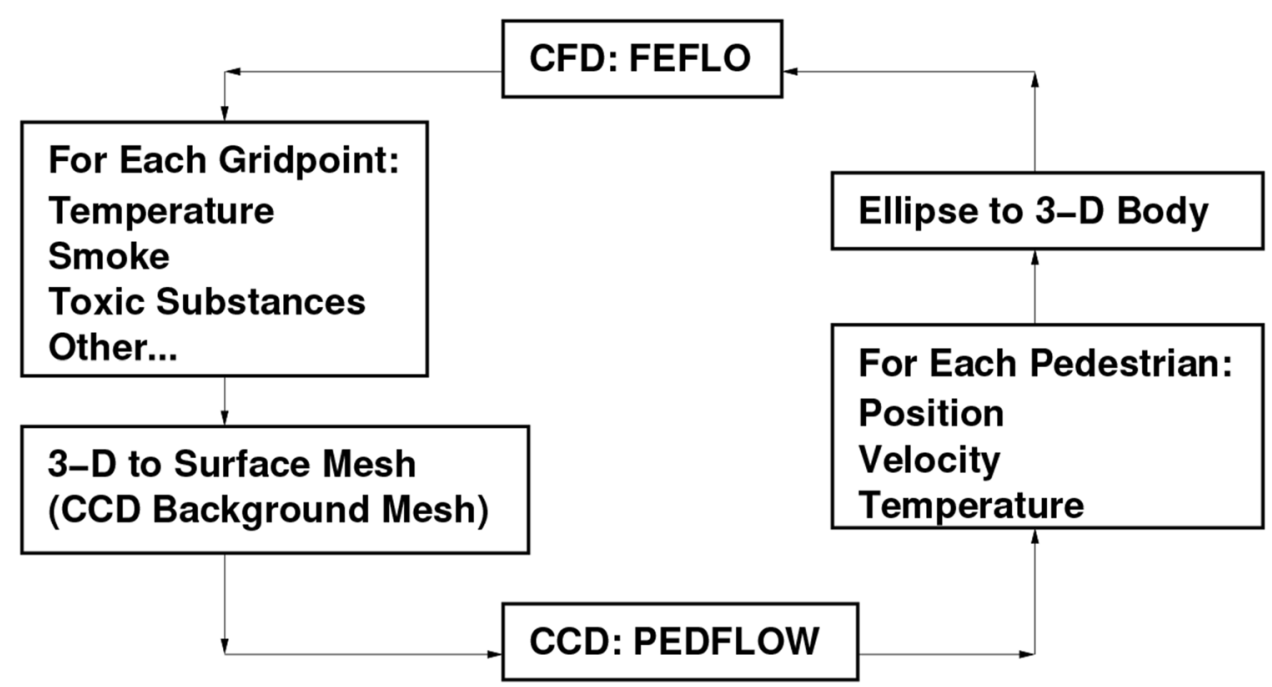


solvers are very small, so that possible coupling errors are negligible. PEDFLOW typically runs with fixed timesteps of $\Delta t_{p}=0.05 \mathrm{~s}$, while the timestep chosen by FEFLO depends on mesh size and velocity. Should the timestep of FEFLO be less than the default value for PEDFLOW, then PEDFLOW automatically reduces its timestep to be the same as FEFLO. During the course of many cases run, we have never encountered any stability problems with this loose coupling and timestepping strategy.

\section{Simplified Coupling Methodology}

As seen before, the pathogen load contained in small droplets follows an advection-diffusion equation of the form:

$c_{, t}+\mathbf{v} \cdot \nabla c=\nabla \cdot k_{c} \nabla c+s_{c}$,

is required, where $c, k_{c}$ denote the concentration and diffusivity of the pathogen, and $s_{c}$ is the source (or sink) term. Given that the airflow occurs in three dimensions with moving pedestrians, this equation needs to be be solved together with the flowfield for the complete three-dimensional space where viral loads are sought. The high cost of these simulations could render this approach impractical for statistical simulations where thousands of instantiations are required. If computer resources are limited, and alternative, simpler model can be used for the propagation of viral loads in space and time. The model assumes that natural convection and/or ventilation systems continually remove viruses in the vertical direction, and that horizontal advection is limited. This simplification is certainly justifiable for crowded situations, and for typical heat/ventilation and air conditioning (HVAC) systems where a strong horizontal flow would be uncomfortable. This leads to an equation of the form:

$c_{, t}=-\lambda c+s_{c}$

where $\lambda$ lumps together the diffusion and advection in the vertical directions.

\subsection{Placement of Pathogen Loads in Space}

A background grid is used for the placement of geographical information in PEDFLOW. The same grid can be used to track pathogen concentrations. As infected pedestrians move though this grid, they exhale pathogen loads-either through sneezing, coughing, shouting or talking. These pathogen loads are added to the concentration $c$ on the background grid.

\subsection{Generation of Pathogen Loads}

Pathogen loads are generated whenever an infected pedestrian exhales, either violently in bursts (e.g. sneezing, coughing, shouting), or continuously (e.g. loud talking). The amount of viral load can vary widely depending on the mode, the state of infection of the pedestrian, and many other factors (the term 'superspreaders' has been used in the medical literature). In the present model, the viral load is deposited at the location of the individual whenever an infected pedestrian exhales. The direction and reach of the viral load depends on the mode: sneezing and coughing have a reach of up to $1.5 \mathrm{~m}$, while loud talking is less than $0.5 \mathrm{~m}$.

\subsection{Inhalation of Pathogen Loads}

As pedestrians walk or run through the clouds of viral loads, they inhale a certain amount of viruses. Given the local concentration of viral load $c$ and the breathing rate of a pedestrian, the total number of viruses inhaled can be integrated in time. The assumption is made that once the inhaled viral load reaches the infectious dose, the pedestrian is considered infected.

\section{Examples}

In the sequel, we show 5 examples of different situations and/or mitigation options. We remark that these are by no means exhaustive or unique: the simulation of aerosol transmission via high-fidelity CFD techniques has received considerable attention in recent years, and has been carried out with commercial and open source software worldwide (see, e.g. [1, 21, 22, 40, 68, 79, 80, 109, 116]). The CFD code used is FEFLO, which was validated for the class of problems considered here over many years $[12,13,14,81$, 91, 92]. Unless otherwise noted, the particles have been coloured according to the logarithm of the diameter, with red colours representing the largest and blue the smallest particles.

\subsection{Sneezing in Subway Car}

One of the obvious vectors for pathogen contamination and spread is mass transport. Passengers are in very close proximity, and airflow may be such that considerable mixing occurs. This prompted the analysis of sneezing in a subway car. The flow enters through two parallel slits in the ceiling, and exits through the ceiling at both ends of the car. The geometry was given from a detailed STL triangulation [31]. The passengers were placed randomly in the car, and an immersed technique was used for them. The mesh had approximately $10.01 \times 10^{6}$ elements. As expected, the 
flowfield is highly turbulent. Figure $7 \mathrm{a}, \mathrm{b}$ shows a snapshot in time. The distribution of particles following a sneeze in the middle of the car but facing to one end can be discerned from Fig. 7c-f. One can see that the large (red) particles follow a ballistic path and fall to the ground. This 'ballistic phase' ends at about $t=1 \mathrm{~s}$. The (green) particles of size $d=0.1 \mathrm{~mm}$ are quickly stopped by the air, and then sink slowly towards the floor in close proximity to the individual sneezing. The even smaller (cyan, blue) particles rise with the cloud of warmer air exhaled by the sneezing individual, and disperse much further at later times.

\subsection{Sneezing in an Airplane Cabin}

The flow in airplane cabins has been extensively discussed by the media thoughout the Covid-19 pandemic. Given that the air in planes is renewed much more often than air-conditioned buildings (one exchange every 2 min versus one exhange every $12-15 \mathrm{~min}$ ) one would assume airplanes to be much safer. Indeed, influenza studies have shown that the 'radius of transmission' is limited to 2-3 rows, with large droplet to surface to hand and then nose/eyes/mouth being the most common route of infection. Nevertheless, there could be regions of stagnant flow that would be condusive to pathogen transmission. This prompted the analysis of cabin flow and sneezing in a B-737-500. The geometry and passenger configuration can be discerned from Fig. 8a.

Unlike older models, the flow in this cabin enters through two parallel slits in the ceiling close to the windows, moves towards the center, and then exits through the holes in the floor close below the windows. The geometry was given from a detailed STL triangulation [31]. The mesh had approximately $890 \times 10^{6}$ elements. The run was carried out to $1 \mathrm{~min}$ of physical time, requiring approximately $24 \mathrm{~h}$ on close to 8000 cores. As expected, the flowfield is highly turbulent. Figure $8 \mathrm{~b}$ shows a snapshot. One can see that at the level of passenger heads the flow is a comfortable $20-30 \mathrm{~cm} / \mathrm{s}$, not too strong, but giving the feeling of an air-conditioned environment. Different 'sneezing positions' were considered, and only the smaller particles were considered. Figure $8 \mathrm{c}-\mathrm{f}$ shows the movement and extent of the 'sneezing clouds' that originated at different positions. Each of these clouds is coloured with a unique colour. One can see that the clouds remain bounded to $2-3$ rows/seats in every direction. This is qualitatively in line with studies of influenza and Cov-19 transmission in airplanes [43, 44, 63]. Note that the age of air (shown in Fig. 8g) at the level of the head of the passengers is less than $50 \mathrm{~s}$. The higher times seen at both ends of the cabin are an artifice of boundary conditions: the outflow conditions in these zones were unknown, and therefore no outflow was considered.

\subsection{Sneezing in Classroom/UV Radiation}

The flow in classrooms has been a source of great concern thoughout the Covid-19 pandemic. This example assesses the effectivity of high-level UV radiation for a particular classroom. The geometry is shown in Fig. 9a. Three UV lamps were placed at the near, far and window sides of the room. The UV radiation is limited to a narrow zone above $2.1 \mathrm{~m}$ which is shown as a transparent cloud on the right half of Fig. 9a. The air enters the room through the four HVAC entries at the ceiling, and exits through the opening on the right of the teacher. As is the case with all (effective) HVACs, the flowfield is highly transient and turbulent. The mesh had approximately $13.82 \times 10^{6}$ elements. The case was run to $900 \mathrm{~s}$ (i.e. $15 \mathrm{~min}$ ) of real time. The particles that became deactivated due to sufficient exposure to UV radiation are not shown. As one can see from Fig. 9c, d, the placement of these high-powered UV lamps has a beneficial effect on pathogen mitigation.

\subsection{Subway Train Station}

This example considers a subway train station and a COVID19 scenario. The length of the domain is approximately $200 \mathrm{~m}$ and two levels are considered. Pedestrians enter the train station randomly (but according to measured data) from the outside and according to train schedules, and exit randomly (but according to measured data) through the entrances or into the trains according to train schedules. The geometry may be discerned from the background grid used both for PEDFLOW and the accumulation of viral loads from Fig. 10a, b. The case was simulated for $600 \mathrm{~s}$ with PEDFLOW. During this time approximately 1800 pedestrians passed through the station. The desired average velocity of pedestrians is $1.2 \mathrm{~m}$, but escalators impose their own velocities, and proper modifications are considered when pedestrians are using stairs [55]. In COVID-19 a substantial fraction of transmission arises from pre-symptomatic and asymptomatic persons, estimated at between 6 and 44\%, albeit with much uncertainty to these numbers [113]. Furthermore, the viral load is highest around the time of onset of symptoms and declines subsequently [113]. Consequently, we can expect that infective persons would sneeze less often than in a disease where infectivity is high after symptom development, and that virus shedding would also be lower in a sneeze. Therefore, the viral transmission parameters were set as follows:

- Percentage of infected pedestrians: $5 \%$;

- Average time between sneezing: $120 \mathrm{~s}$;

- Average viral load shed per sneeze: 0.5 ;

- Radius of initial dispersal: $0.5 \mathrm{~m}$;

- Decay time for viral load: $10.0 \mathrm{~s}$; 
Fig. 7 a, b Subway car:

magnitude of velocity field at

$\mathrm{T}=27.5 \mathrm{~s}(\mathrm{~m} / \mathrm{s})$. c-f Subway

car: particle distribution in time
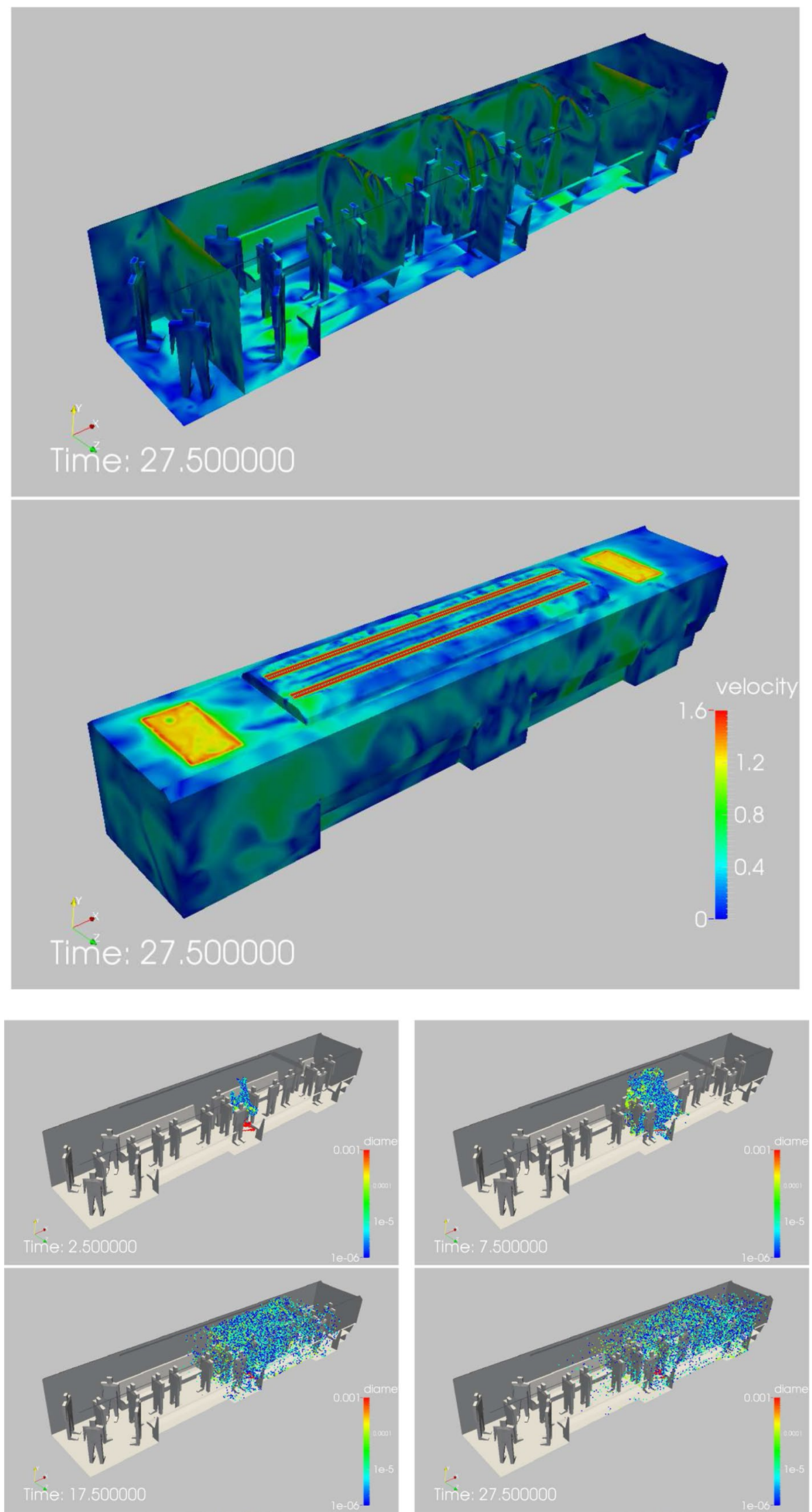


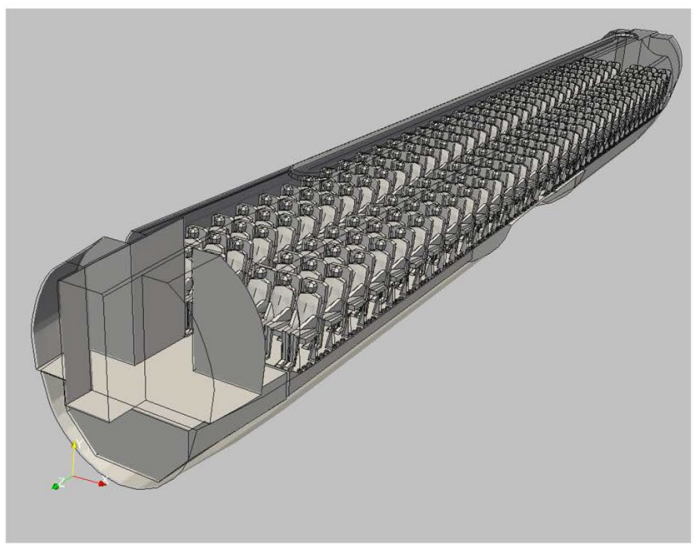

a Airplane Cabin: Geometry and Seating Arrangement

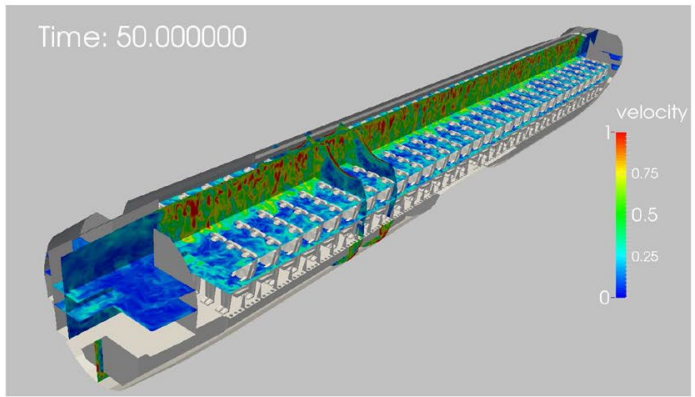

b Airplane Cabin: Snapshot of Magnitude of Velocity Field [m/sec]

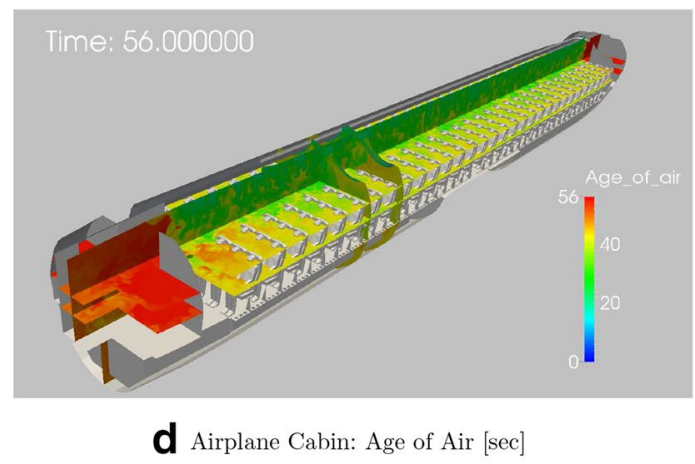

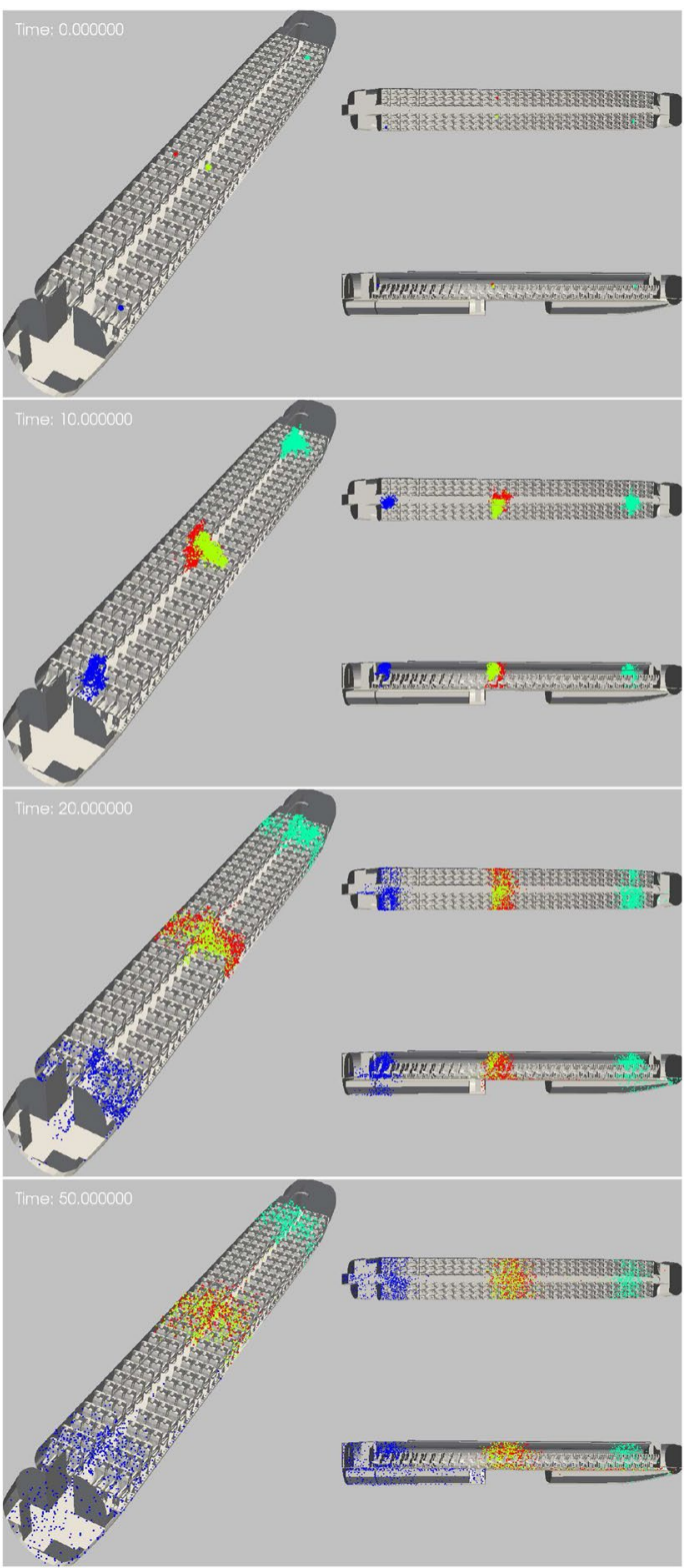

C Airplane Cabin: Sneezing in Different Locations

Fig. 8 a Airplane cabin: geometry and seating arrangement. b Airplane cabin: snapshot of magnitude of velocity field (m/s). c Airplane cabin: sneezing in different locations. d Airplane cabin: age of air (s)

- Threshold for getting infected: 0.4 .

Figure 10c-e shows different snapshots in time, where the viral load is superposed to the pedestrians. The blue, green and red squares denote pedestrians that are healthy, entering the domain infected and being infected respectively. In order to gain some statistical insight into the model, 1000 runs were conducted. Figure $10 \mathrm{f}$ shows the probability of the number of infected people. The 'reproductive number' or 'infection rate' is close to $R 0=1.0$, i.e. each infected pedestrian infects on average one other pedestrian. 

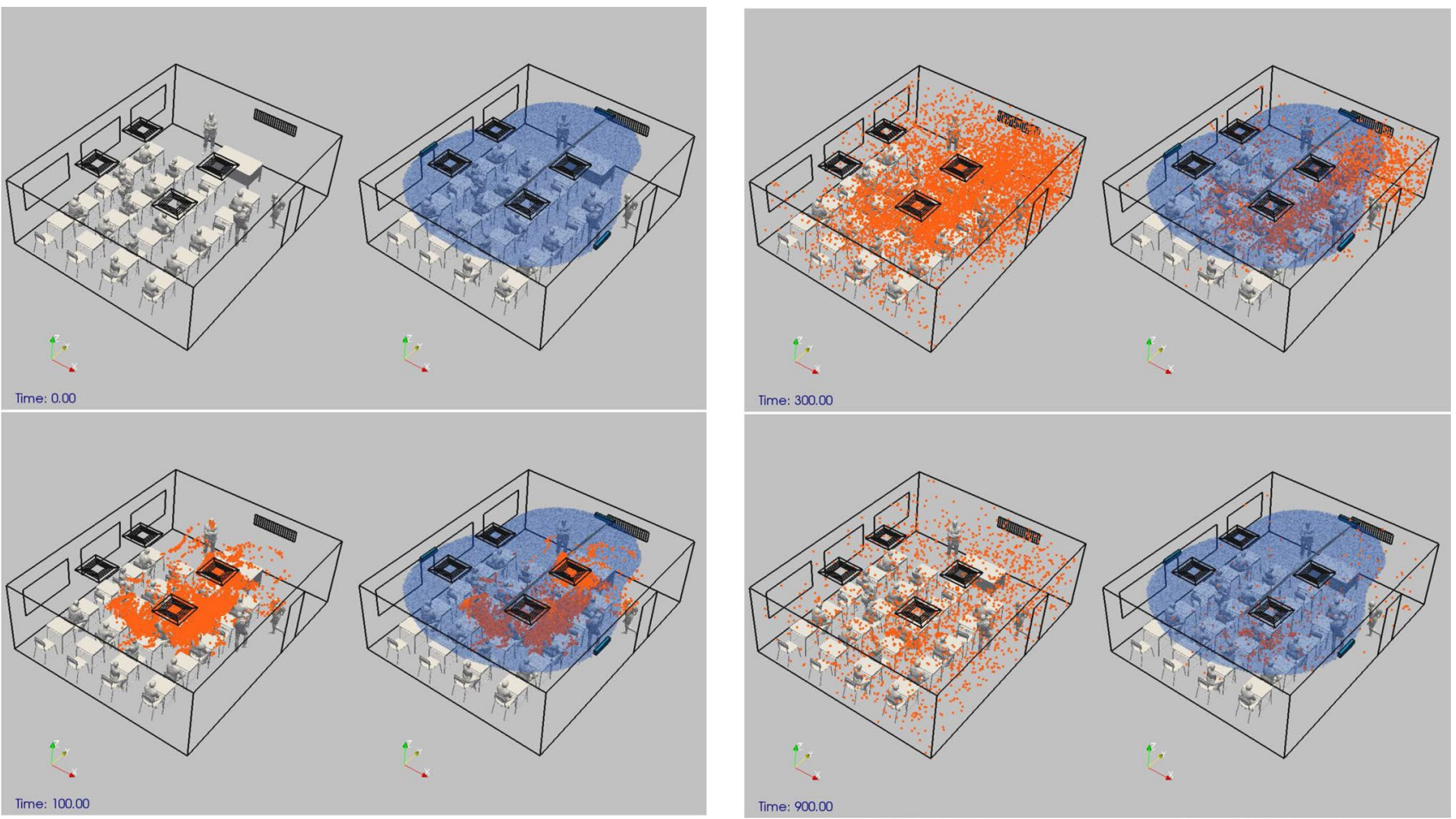

Fig. 9 a, b Classroom: active pathogens without (left) and with (right) UV. c, d Classroom: active pathogens without (left) and with (right) UV

\subsection{Corridor With Pedestrians}

This example considers the corridor of $10.0 \mathrm{~m} \times 2.0 \mathrm{~m} \times 2.5 \mathrm{~m}$ shown in Fig. 11a. Both entry and exit sides have two doors each of size $0.8 \mathrm{~m} \times 2.0 \mathrm{~m}$. For climatisation, 4 entry vanes and 1 exit vane are placed in the ceiling. The vertical air velocity for the entry vanes was set to $v_{z}=0.2 \mathrm{~m} / \mathrm{s}$, while the horizontal velocity was set as increasing proportional to the distance of the center of the vane to a maximum of $v_{r}=0.4 \mathrm{~m} / \mathrm{s}$. The CFD mesh had approximately $3.5 \times 10^{6}$ elements, and as can be seen from Fig. 11b, c had a uniform element size. Two streams of pedestrians enter and exit through the doors over time. As stated before, the pedestrian dynamics code, which only 'sees' the floorplan (Fig. 11d) of the problem at hand, computes position, velocity and orientation of the pedestrians, produces a tetrahedral mesh for each pedestrian (Fig. 11e), and sends this information to the transfer library. This information is then passed on to the flow solver, which treats the pedestrians via the immersed body approach in the flowfield. Should there be smoke, pollutants or pathogens in the flowfield, this information is passed back to the pedestrian dynamics code, which interpolates it at the height of pedestrians in order to update inhalation, intoxication and infection information.

This simulation was run in 3 phases:
- Phase 1: Every code is run independently until the flow reaches a quasi-steady state and the pedestrian streams have formed; for the present case this took $20 \mathrm{~s}$ of physical time;

- Phase 2: The restart files from Phase 1 are taken, and the run continues in fully coupled mode, until a quasi-steady state is reached; for the present case this took $20 \mathrm{~s}$ of physical time;

- Phase 3: The restart files from Phase 2 are taken, and the run continues in fully coupled mode imposing the boundary conditions for a sneezing event.

A number of cases were run [79], of which only the one with two pedestrians streams in counterflow mode is shown here in Fig. 11f-h. Immediately after the sneeze (Fig. 11f), all particles are in the air and the 'cloud of pathogens' is limited to the immediate vicinity of the pedestrian that sneezed. After $2.0 \mathrm{~s}$ (Fig. 11g), the large (red) droplets have fallen to the ground, while the "cloud of pathogens in small particles' has extended considerably and is beginning to show the effects of the counterflow. At $10.0 \mathrm{~s}$ (Fig. 11h), the cloud has propagated throughout the corridor. The different velocities between walking pedestrians, and in particular counterflows as the one shown, lead to large-scale turbulent mixing, enhancing the spread of pathogens emanating from infected victims. 


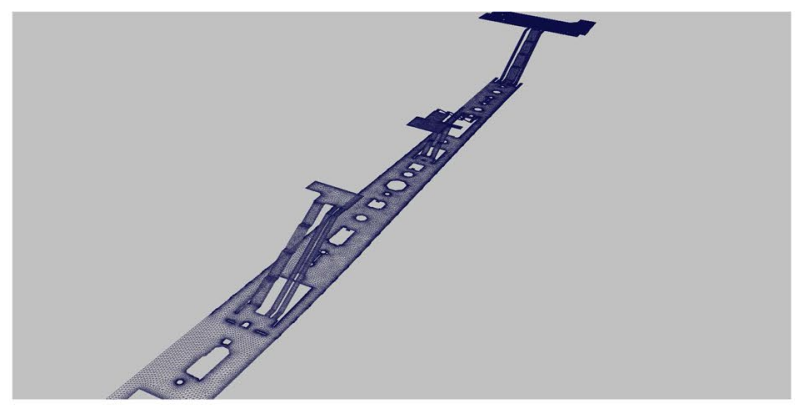

a Train Station: Geometry/Background Mesh

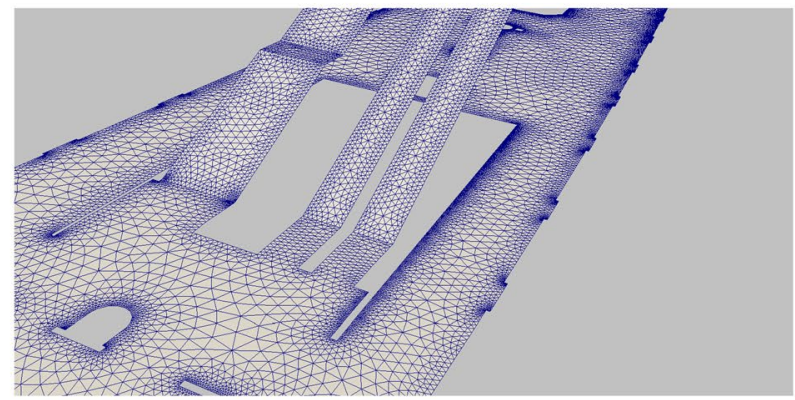

b Train Station: Geometry/Background Mesh (Detail)

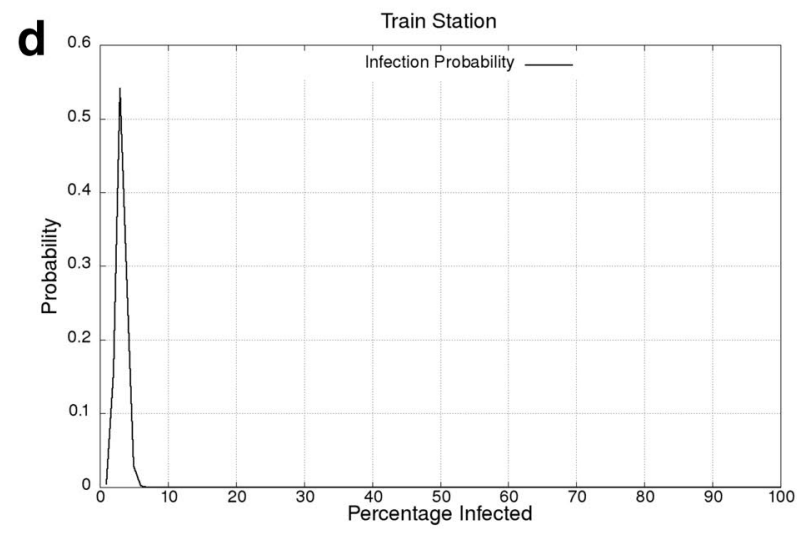

Fig. 10 a Train station: geometry/background mesh. b Train station: geometry/background mesh (detail). c Train station: sneezing and infection. d Train station: probability of people infected

\section{Conclusions and Outlook}

An overview of high-fidelity modeling of pathogen propagation, transmission and mitigation in the built environment has been given. In order to derive the required physical and numerical models, the current understanding of pathogen, and in particular virus transmission and mitigation was summarized. The ordinary and partial differential equations that describe the flow, the particles and possibly the UV radiation loads in rooms or HVAC ducts were given, as well as proper numerical methods to solve them in an expedient way. The attention then turned to the motion of pedestrians, as well as proper ways to couple computational fluid dynamics (CFD) and computational crowd dynamics (CCD)

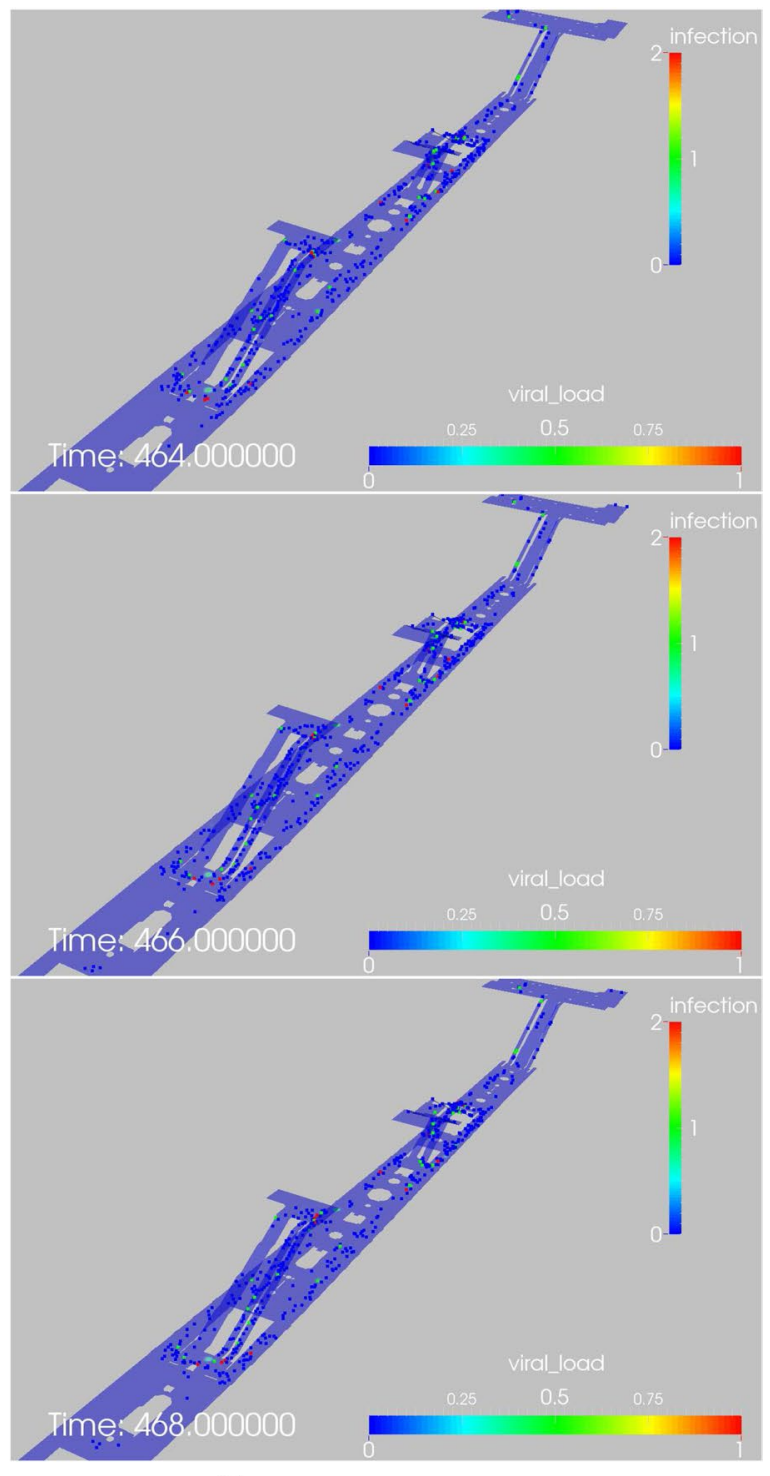

C Train Station: Sneezing and Infection 


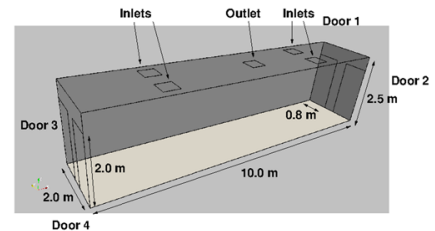

a Simple Channel: Geometry and Boundary Conditions

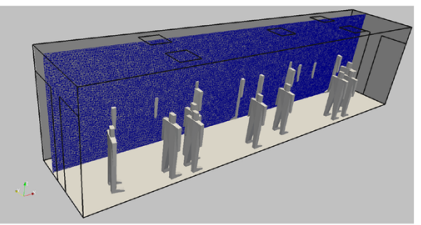

b Simple Channel: Y-Plane-Cut of CFD Mesh

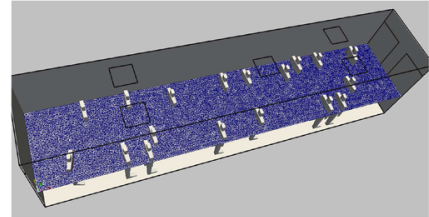

C Simple Channel: Z-Plane-Cut of CFD Mesh

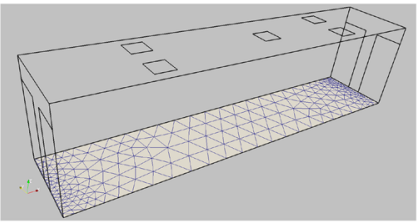

d Simple Channel: CCD Background Mesh

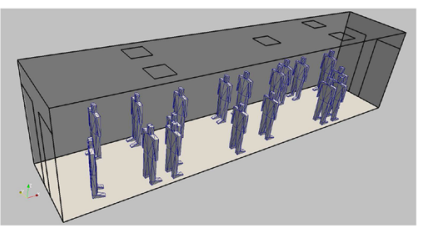

e Simple Channel: Surface of Tetrahedral Element for Immersed BC of Pedestrians in CFD Mesh
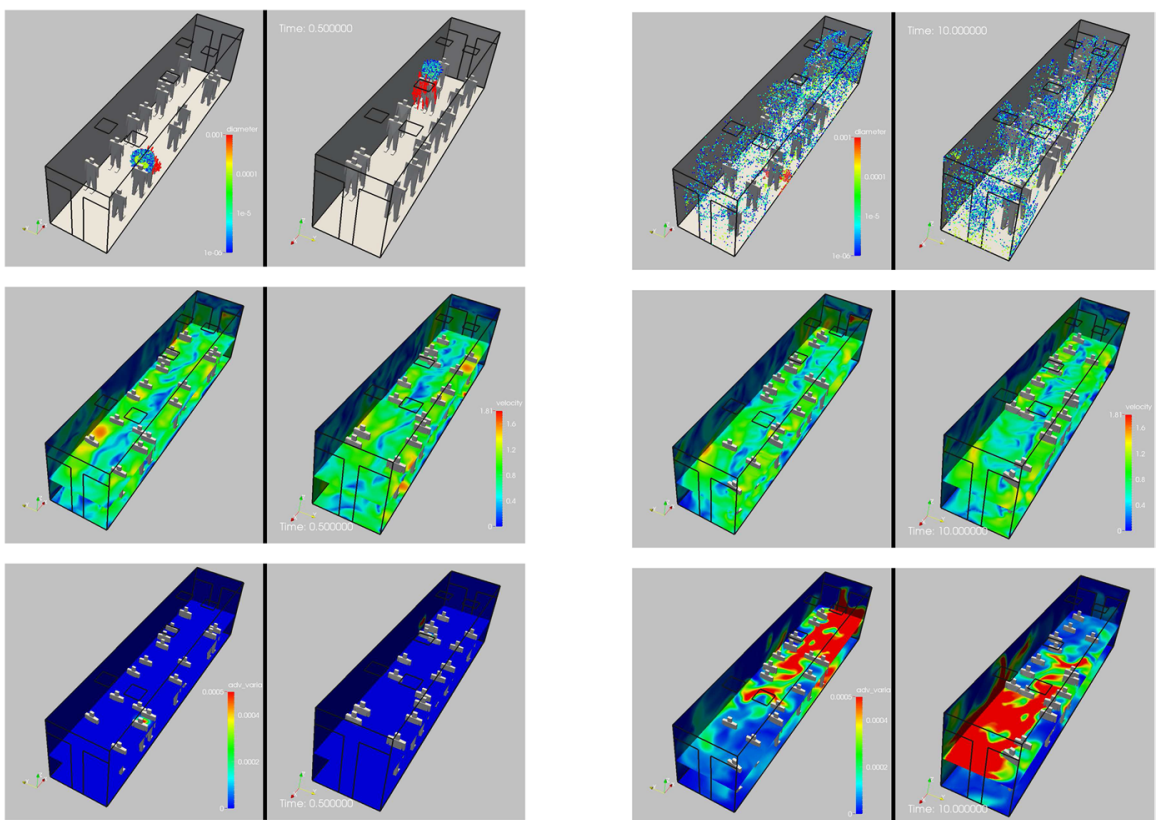

f Counterflow Movement: Solution at $t=0.50 \mathrm{sec}$

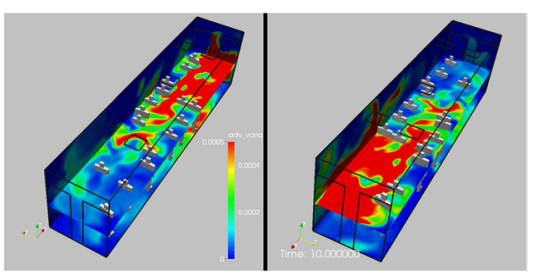

h Counterflow Movement: Solution at $t=10.00 \mathrm{sec}$
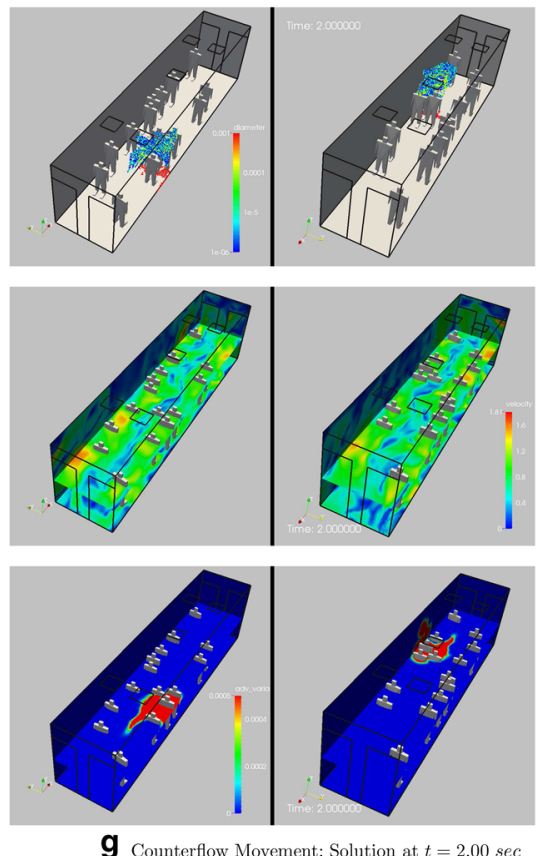

Fig. 11 a Simple channel: geometry and boundary conditions. b Simple channel: Y-plane-cut of CFD mesh. c Simple channel: Z-plane-cut of CFD mesh. d Simple channel: CCD background mesh. e Simple channel: surface of tetrahedral elements for

immersed BC of pedestrians in CFD mesh. f Counterflow movement: solution at $t=0.50 \mathrm{~s}$. $g$ Counterflow movement: solution at $t=2.00 \mathrm{~s}$. h Counterflow movement: solution at $t=10.00 \mathrm{~s}$

Furthermore, even though the basic physical phenomena and the partial and ordinary differential equations describing them have been known for over a century, and solvers have advanced considerably over the last four decades, a 
vigorous experimental program is needed to complement and validate the numerical methods, and to establish firm 'best practice' guidelines.

Acknowledgements It is a pleasure the acknowledge the help of Mika Gröndahl from the graphics department of the New York Times for the detailed STL models and throroughly researched boundary conditions of the subway train car and the airplane cabin. These runs would have been either impossible or meaningless without his efforts. H. Antil is partially supported by NSF Grants DMS-1818772, DMS-1913004, the Air Force Office of Scientific Research (AFOSR) under Award No.: FA9550-19-1-0036, and Department of Navy, Naval PostGraduate School under Award No.: N00244-20-1-0005. E. Oñate and S. Idelsohn acknowledge financial support from the project PARAFLUIDS (PID2019-104528RB-I00) of the National Research Plan of the Spanish Government, from the CERCA programme of the Generalitat de Catalunya, and from the Spanish Ministry of Economy and Competitiveness, through the 'Severo Ochoa Programme for Centres of Excellence in R\&D' (CEX2018-000797-S).

\section{References}

1. Abuhegazy M, Talaat K, Anderoglu O, Poroseva SV (2020) Numerical investigation of aerosol transport in a classroom with relevance to COVID-19. Phys Fluids. https://doi.org/10.1063/5. 0029118

2. Armstrong TW, Haas CN (2007) A quantitative microbial risk assessment model for legionnaires' disease: animal model selection and dose-response modeling. Risk Anal 27(6):1581-1596. https://doi.org/10.1111/j.1539-6924.2007.00990.x

3. Asadi S, Bouvier NM, Wexler AS, Ristenpart W (2020) The coronavirus pandemic and aerosols: does COVID-19 transmit via expiratory particles ? Aerosol Sci Technol. https://doi.org/ 10.1080/02786826.2020.1749229

4. Asadi S, Wexler AS, Cappa CD, Barreda S, Bouvier NM, Ristenpart W (2019) Aerosol emission and superemission during human speech increase with voice loudness. Nat Sci Rep 9(1):2348. https://doi.org/10.1038/s41598-019-38808-z

5. Asadi S, Wexler AS, Cappa CD, Barreda S, Bouvier NM, Ristenpart W (2020) Effect of voicing and articulation manner on aerosol particle emission during human speech. PLOS ONE. https://doi.org/10.1371/journal.pone.0227699

6. ASHRAE Position Document on Airborne Infectious Diseases; ASHRAE. American Society of Heating, Refrigerating and AirConditioning Engineers (2020). https://www.ashrae.org/file\%20 library/about/position\%20documents/airborne-infectious-diseases.pdf

7. Blue VJ, Adler JL (2002) Flow capacities from cellular automata modeling of proportional splits of pedestrians by direction. In: Schreckenberg M, Sharma SD (eds) Pedestrian and evacuation dynamics. Springer, Berlin, pp 115-122

8. Blue VJ, Adler JL (1998) Emergent fundamental pedestrian flows from cellular automata microsimulation. Transp Res Rec 1644:29-36

9. Boris JP, Grinstein FF, Oran ES, Kolbe RJ (1992) New insights into large eddy simulation. Fluid Dyn Res 10:199-228

10. Brouwer AF, Weir MH, Eisenberg MC, Meza R, Eisenberg JNS (2017) Dose-response relationships for environmentally mediated infectious disease transmission models. PLoS Comput Biol. https://doi.org/10.1371/journal.pcbi.1005481
11. Bungartz H-J, Schäfer M (eds.) (2006) Fluid-structure interaction. Springer Lecture Notes in Computational Science and Engineering. Springer

12. Camelli F, Löhner R (2004) Assessing maximum possible damage for contaminant release events. Eng Comput 21(7):748-760

13. Camelli F, Löhner R (2006) VLES study of flow and dispersion patterns in heterogeneous urban areas. AIAA-06-1419

14. Camelli F, Löhner R, Sandberg WC, Ramamurti R (2004) VLES study of ship stack gas dynamics. AIAA-04-0072

15. Cebral JR, Löhner R (2005) On the loose coupling of implicit time-marching codes. AIAA-05-1093

16. Chang K (2020) Scientists consider indoor ultraviolet light to zap coronavirus in the air. The New York Times ISSN 0362-4331

17. Chao C, Wan MP, Morawska L, Johnson G, Graham R, Ristovski Z, Hargreaves M, Mengersen K, Kerrie L, Steve C, Li Y, Xie X, Katoshevski S (2009) Characterization of expiration air jets and droplet size distributions immediately at the mouth opening. J Aerosol Sci 40(2):122-133

18. Clift R, Grace JR, Weber ME (1978) Bubbles, drops and particles. Academic Press, New York

19. Courty N, Musse S (2005) Simulation of large crowds including gaseous phenomena. In: Proceedings of IEEE computer graphics international 2005. New York, pp 206-212

20. Curtis S, Manocha D (2012) Pedestrian simulation using geometric reasoning in velocity space. In: Weidmann U, Kirsch U, Schreckenberg M (eds) Pedestrian and evacuation dynamics. Springer, Heidelberg

21. Dbouk T, Drikakis D (2020) On coughing and airborne droplet transmission to humans. Phys Fluids

22. Dbouk T, Drikakis D (2020) On respiratory droplets and face masks. Phys Fluids. https://doi.org/10.1063/5.0015044

23. Dietz L, Horve PF, Coil DA, Fretz M, Eisen JA, van den Wymelenberg L (2020) Novel coronavirus (COVID-19) pandemic: built environment considerations to reduce transmission. mSystems 5:e00245. https://doi.org/10.1128/mSystems.00245-20

24. Dijkstra J, Jesurun J, Timmermans H (2002) A multi-agent cellular automata model of pedestrian movement. In: Schreckenberg M, Sharma SD (eds) Pedestrian and Evacuation Dynamics. Springer, Berlin, pp 173-180

25. Fabian P, McDevitt JJ, Dehaan WH, Fung ROP, Cowling BJ, Chan $\mathrm{KH}$, et al (2008) Influenza virus in human exhaled breath: an observational study. PLoS ONE 3:e2691

26. Favre J, Löhner R (1994) Raytracing with a space-filling finite element mesh. Int J Numer Methods Eng 37(20):3571-3580

27. Franz DR, Jahrling PB, Friedlander AM, et al (1997) Clinical recognition and management of patients exposed to biological warfare agents. JAMA 278:399e411

28. Fruin JJ (1971) Pedestrian Planning and Design. Metropolitan Association of Urban Designers and Environmental Planners, New York

29. Fureby C, Grinstein F (1999) Monotonically integrated large eddy simulation of free shear flows. AIAA J 37(5):544-556

30. Grinstein FF, Fureby C (2002) Recent progress on MILES for high-Reynolds-number flows. J Fluids Eng 124:848-861

31. Gröndahl M, Goldbaum Ch, White J (2020) What happens to viral particles on the subway. New York Times, August 10; see also https://www.nytimes.com/interactive/2020/08/10/nyregion/ nyc-subway-coronavirus.html

32. Guidance for Cleaning and Disinfecting Public Spaces, Workplaces, Businesses, Schools, and Homes. CDC paperhttps://www. cdc.gov/coronavirus/2019-ncov/community/pdf/Reopening _ America_Guidance.pdf (2020)

33. Gupta JK, Lin C-H, Chen Q (2009) Flow dynamics and characterization of a cough. Indoor Air 19:517-525 
34. Gupta JK, Lin C-H, Chen Q (2010) Characterizing exhaled airflow from breathing and talking. Indoor Air 20:31-39

35. Gupta JK, Lin C-H, Chen Q (2011) Inhalation of expiratory droplets in aircraft cabins. Indoor Air 21:341-350. https://doi.org/10. 1111/j.1600-0668.2011.00709.x

36. Gupta JK, Lin C-H, Chen Q (2011) Transport of expiratory droplets in an aircraft cabin. Indoor Air 21:3-11

37. Gupta JK, Lin C-H, Chen Q (2012) Risk assessment of airborne infectious diseases in aircraft cabins. Indoor Air 22(5):388-395

38. Guy SJ, Chhugani J, Curtis S, Dubey P, Lin M, Manocha D (2010) PLEdestrians: a least-effort approach to crowd simulation. In: Eurographics/ACM SIGGRAPH symposium on computer animation, Madrid, Spain

39. Guy SJ, Chhugani J, Kim C, Satish N, Lin M, Manocha D, Dubey P (2009) ClearPath: highly parallel collision avoidance for multiagent simulation. In: Fellner D, Spencer S (eds) Proceedings of ACM SIGGRAPH/Eurographics symposium on computer animation. Association of Computing Machinery, New York, pp 177-187

40. Halloran SK, Wexler AS, Ristenpart WD (2012) A comprehensive breath plume model for disease transmission via expiratory aerosols. PLoS ONE. https://doi.org/10.1371/journal.pone.00370 88

41. Helbing D, Molnar P (1995) Social force model for pedestrian dynamics. Phys Rev E 51:4282-4286

42. Helbing D, Farkas IJ, Molnár P, Vicsek T (2002) Simulation of pedestrian crowds in normal and evacuation situations. In: Schreckenberg M, Sharma SD (eds) Pedestrian and evacuation dynamics. Springer, Berlin, pp 21-58

43. Hertzberg VS, Weiss $\mathrm{H}$ (2016) On the 2-row rule for infectious disease transmission on aircraft. Ann Glob Health 82(5):819823. https://doi.org/10.1016/j.aogh.2016.06.003

44. Hertzberg VS, Weiss H, Elon L, Si W, Norris SL (2018) FlyHealthy research team-behaviors, movements, and transmission of droplet-mediated respiratory diseases during transcontinental airline flights. Proc Natl Acad Sci USA 115(14):3623-3627. https://doi.org/10.1073/pnas.1711611115

45. https://en.wikipedia.org/wiki/Ultraviolet_germicidal_irradiation (2020)

46. https://www.cdc.gov/coronavirus/2019-ncov/hcp/infection-contr ol-recommendations.html

47. https://www.cdc.gov/coronavirus/2019-ncov/symptoms-testing/ symptoms.html

48. https://www.cdc.gov/infectioncontrol/guidelines/environmental/ background/air.html

49. https://www.webmd.com/lung/how-long-covid-19-lives-on-surfa ces (2020)

50. Hughes RL (2003) The flow of human crowds. Annu Rev Fluid Mech 35:169-182

51. Idelsohn SR, Nigro N, Larreteguy A, Gimenez JM, Ryshakov P (2019) A pseudo-DNS method for the simulation of incompressible fluid flows with instabilities at different scales. Int J Comp Particle Mech. https://doi.org/10.1007/s40571-019-00264-x

52. Ip M, Tang JW, Hui DSC, Wong ALN, Chan MTV, Joynt GM, So ATP, Hall SD, Chan PKS, Sung JJY (2007) Airflow and droplet spreading around oxygen masks: a simulation model for infection control research. AJIC 35(10):684-689

53. Isenhour M (2016) Simulating occupant response to emergency situations. George Mason University, Fairfax (PhD Thesis)

54. Isenhour M, Löhner R (2014) Verification of a pedestrian simulation tool using the NIST recommended test cases; the conference in pedestrian and evacuation dynamics 2014 (PED2014). Transp Res Proc 2:237-245

55. Isenhour M, Löhner R (2014) Verification of a pedestrian simulation tool using the NIST stairwell evacuation data; the conference in pedestrian and evacuation dynamics 2014 (PED2014). Transp Res Proc 2:739-744

56. Isenhour M, Löhner R (2016) Pedestrian speed on stairs: a mathematical model based on empirical analysis for use in computer simulations In: Song, W Ma J, Fu L (eds) Proceedings of pedestrian and evacuation dynamics 2016 (PED 2016). University of Science and Technology Press, Hefei, China, pp 529-533

57. Isenhour M, Löhner R (2016) Validation data from the evacuation of a student center. In: Song W, Ma J, Fu L (eds) Proceedings of pedestrian and evacuation dynamics 2016 (PED 2016). University of Science and Technology Press, Hefei, China, pp. 472-479

58. Jameson A, Schmidt W, Turkel E (1981) Numerical solution of the euler equations by finite volume methods using Runge-Kutta time-stepping schemes. AIAA-81-1259

59. Johnson GR, Morawska L, Ristovski ZD, Hargreaves M, Mengersen K, Chao CYH, Wan MP, Li Y, Xie X, Katoshevski D, Corbette S (2011) Modality of human expired aerosol size distributions. J Aerosol Sci 42:839-851

60. Kampf G, Todt D, Pfaender S, Steinmann E (2020) Persistence of coronaviruses on inanimate surfaces and their inactivation with biocidal agents. J Hosp Infect 104(3):246-251. https://doi.org/ 10.1016/j.jhin.2020.01.022

61. Karmakharm T, Richmond P, Romano DM (2010) Agent-based large scale simulation of pedestrians with adaptive realistic navigation vector fields. In: Collomosse J, Grimstead I (eds) EG UK theory and practice of computer graphics 2010

62. Kessel A, Klüpfel H, Wahle J, Schreckenberg M (2002) Microscopic simulation of pedestrian crowd motion. In: Schreckenberg M, Sharma SD (eds) Pedestrian and evacuation dynamics. Springer, pp 193-202

63. Khanh NC, Thai PQ, Quach HL, Thi NH, Dinh PC, Duong TN, Mai LTQ, Nghia ND, Tu TA, QuangN N, Quang TD, Nguyen TT, Vogt F, Anh DD (2020) Transmission of SARS-CoV 2 during long-haul flight. Emerg Infect Dis 26(11):2617-2624. https:// doi.org/10.3201/eid2611.203299

64. Klüpfel HL (2003) A cellular automation model for crowd movement and egress simulation. Ph.D. Dissertation, Falkutät 4, Univ. Duisburg-Essen

65. Lakoba TI, Kaup DJ, Finkelstein NM (2005) Modifications of the Helbing-Molnár-Farkas-Vicsek social force model for pedestrian evolution. Simulation 81, 339 (2005)

66. Langston PA, Masling R, Asmar BN (2006) Crowd dynamics discrete element multi-circle model. Saf Sci 44:395-417

67. Li Y et al (2007) Role of ventilation in airborne transmission of infectious agents in the built environment-a multidisciplinary systematic review. Indoor Air 17:2-18

68. Li H, Leong FY, Xu G, Ge Z, Kang CW, Lim KH (2020) Dispersion of evaporating cough droplets in tropical outdoor environment. Phys Fluids. https://doi.org/10.1063/5.0026360

69. Lindsley WG, Blachere FM, Thewlis RE, Vishnu A, Davis KA, Cao G, et al (2010) Measurements of airborne influenza virus in aerosol particles from human coughs. PLoS ONE 5:e15100

70. Lindsley WG, Pearce TA, Hudnall JB, Davis KA, Davis SM, Fisher MA et al (2012) Quantity and size distribution of coughgenerated aerosol particles produced by influenza patients during and after illness. J Occup Environ Hyg 9:443-449

71. Löhner R (2011) Coupling several CFD and CSD codes in one application in special edition. Int J Multiphys 3:1-16

72. Löhner R (2004) Multistage explicit advective prediction for projection-type incompressible flow solvers. J Comput Phys 195:143-152

73. Löhner R (2008) Applied CFD techniques, 2nd edn. Wiley, London

74. Löhner R (2010) On the modeling of pedestrian motion. Appl Math Model 34(2):366-382 
75. Löhner R, Ambrosiano J (1990) A vectorized particle tracer for unstructured grids. J Comput Phys 91(1):22-31

76. Löhner R, Cebral JR, Camelli FF, Appanaboyina S, Baum JD, Mestreau EL, Soto O (2008) Adaptive embedded and immersed unstructured grid techniques. Comput Methods Appl Mech Eng 197:2173-2197

77. Löhner R, Camelli F, Baum JD, Togashi F, Soto O (2014) On mesh-particle techniques. Comp Part Mech 1:199-209

78. Löhner R, Baqui M, Haug E, Muhamad B (2016) Realtime micro-modelling of a million pedestrians. Eng Comput 33(1):217-237

79. Löhner R, Antil H (2020) High fidelity modeling of aerosol pathogen propagation in built environments with moving pedestrians. Int J Numer Methods Biomed Eng. https://doi.org/10.1002/cnm. 3428arXiv:2009.03365 [physics.soc-ph]

80. Löhner R, Antil H, Idelsohn S, Oñate E (2020) Detailed simulation of viral propagation in the built environment. Comput Mech. https://doi.org/10.1007/s00466-020-01881-7. arXiv:2006.13792 [physics.soc-ph]

81. Löhner R, Camelli F (2016) Tightly coupled computational fluid and crowd dynamics. In: Song W, Ma J, Fu L (eds) Proceedings of pedestrian and evacuation dynamics 2016 (PED 2016). University of Science and Technology Press, Hefei, China, pp 505-509

82. Löhner R, Yang C, Cebral JR, Camelli F, Soto O, Waltz J (2006) Improving the speed and accuracy of projection-type incompressible flow solvers. Comput Methods Appl Mech Eng 195:23-24 (2006)

83. Löhner R, Yang C, Cebral J, Baum JD, Luo H, Pelessone D, Charman C (1995) Fluid-structure interaction using a loose coupling algorithm and adaptive unstructured grids. AIAA-95-2259 [Invited]

84. Loudon RG, Roberts RM (1967) Droplet expulsion from the respiratory tract. Am Rev Respir Dis 95(3):435-442

85. Milton DK, Fabian MP, Cowling BJ, Grantham ML, McDevitt JJ (2013) Influenza virus aerosols in human exhaled breath: particle size, culturability, and effect of surgical masks. PLoS Pathog 9:e1003205

86. Namilae S, Derjany P, Mubayi A, Scotch M, Srinivasan A (2017) Multiscale model for pedestrian and infection dynamics during air travel. Phys Rev E

87. Pelechano N, Badler NI (2006) Modeling crowd and trained leader behavior during building evacuation. IEEE Comput Graph Appl 26(6):80-86

88. Pelechano N, Allbeck J, Badler NI (2008) Virtual crowds: methods, simulation and control. Morgan \& Claypool, San Rafael

89. Predtetschenski WM, Milinski AI (1971) Personenströme in Gebäuden - Berechnungsmethoden für die Projektierung. Verlaggesellschaft Rudolf Müller, Köln-Braunsfeld

90. Quinn MJ, Metoyer RA, Hunter-Zaworski K (2003) Parallel implementation of the social forces model. In: Proceedings 2nd international conference in pedestrian and evacuation dynamics, pp 63-74

91. Ramamurti R, Löhner R (1996) A parallel implicit incompressible flow solver using unstructured meshes. Comput Fluids 5:119-132

92. Ramamurti R, Sandberg WC, Löhner R (1999) Computation of unsteady flow past deforming geometries. Int J Comput Fluid Dyn 13:83-99

93. Schadschneider A (2002) Cellular automaton approach to pedestrian dynamics: theory. In: Schreckenberg M, Sharma SD (eds) Pedestrian and evacuation dynamics. Springer, pp 75-86

94. Schäfer M, Turek S (eds) (2008) Proceedings of international workshop on fluid-structure interaction: theory, numerics and applications, Herrsching (Munich), Germany, Sept 29-Oct 1 (2008)
95. Schlichting H (1979) Boundary layer theory. McGraw-Hill, London

96. Schreckenberg M, Sharma SD (eds) (2002) Pedestrian and evacuation dynamics. Springer, Berlin

97. Sud A, Gayle R, Andersen E, Guy S, Lin Ming, Manocha D (2007) Real-time navigation of independent agents using adaptive roadmaps. In: ACM symposium on virtual reality software and technology

98. Sze To GN, Chao CY (2010) Review and comparison between the Wells-Riley and dose-response approaches to risk assessment of infectious respiratory diseases. Indoor Air 20(1):2-16. https://doi.org/10.1111/j.1600-0668.2009.00621.x

99. Tang JW, Li Y, Eames I, Chan PKS, Ridgway GL (2006) Factors involved in the aerosol transmission of infection and control of ventilation in healthcare premises. J Hosp Infect 64:100-114

100. Tang JW, Noakes CJ, Nielsen PV, Eames I, Nicolle A, Li Y, Settles GS (2011) Observing and quantifying airflows in the infection control of aerosol- and airborne-transmitted diseases: an overview of approaches. J Hosp Infect 77:213-222

101. Tang JW, Nicolle AD, Pantelic J, Koh GC, Wang L, Amin M, Klettner CA, Cheong SKW, Sekhar C, Tham KW (2012) Airflow dynamics of coughing in healthy human volunteers by shadowgraph imaging: an aid to aerosol infection control. PLoS ONE 7(4):e34818. https://doi.org/10.1371/journal.pone.0034818

102. Tang JW, Nicolle AD, Klettner CA, Pantelic J, Wang L, Bin Suhaimi A, Tan AYL, Ong GWX, Su R, Sekhar C, Cheong DKW, Tham KW (2013) Airflow dynamics of human jets: sneezing and breathing - potential sources of infectious aerosols. PLoS ONE 8(4):e59970. https://doi.org/10.1371/journal.pone.0059970

103. Teknomo K, Takeyama Y, Inamura H (2000) Review on microscopic pedestrian simulation model. In: Proceedings of Japan Society of Civil Engineering Conference. Morioka, Japan

104. Teunis PFM, Brienen N, Kretzschmar MEE (2010) High infectivity and pathogenicity of influenza A virus via aerosol and droplet transmission. Epidemics 2:215-222

105. Thalmann D, Musse SR (2007) Crowd simulation. Springer, London

106. Torrens PM (2012) Moving agent pedestrians through space and time. Ann Assoc Am Geogr 102(1):35-66

107. van Doremalen N, Bushmaker T, Morris DH, Holbrook MG, Gamble A, Williamson BN, Tamin A, Harcourt JL, Thornburg NJ, Gerber SI, Lloyd-Smith JO, de Wit E, Munster VJ (2020) Aerosol and surface stability of SARS-CoV-2 as compared with SARS-CoV-1. N Engl J Med 382:1564-1567. https://doi.org/10. 1056/NEJMc2004973

108. Vigueras G, Lozano M, Ordun JM, Grimaldo F (2010) A comparative study of partitioning methods for crowd simulations. Appl Soft Comput 10:225-235

109. Villafruela JM, Olmedo I, Ruiz de Adana M, Mendez C, Nielsen PV (2013) CFD analysis of the human exhalation flow using different boundary conditions and ventilation strategies. Build Environ 62:191-200

110. Vishton PM, Cutting JE (1995) Wayfinding, displacements, and mental maps: velocity fields are not typically used to determine one's aimpoint. J Exp Psychol 21(5):978-995

111. Wei J, Li Y (2016) Airborne spread of infectious agents in the indoor environment. Am J Infect Control 44:S102-S108. https:// doi.org/10.1016/j.ajic.2016.06.003

112. Wells WF (1955) Airborne contagion and air hygiene. An ecological study of droplet infections. Cambridge University Press, Cambridge

113. World Health Organization-Transmission of SARS-CoV-2: implications for infection prevention precautions; Scientific Brief, July 9 (2020). https://www.who.int/news-room/comme ntaries/detail/transmission-of-sars-cov-2-implications-for-infec tion-prevention-precautions 
114. Xie X, Li Y, Chwang ATY, Ho PL, Seto WH (2007) How far droplets can move in indoor environments-revisiting the wells evaporation-falling curve. Indoor Air 17:211-225. https://doi. org/10.1111/j.1600-0668.2006.00469.x

115. Zhang J, Britto D, Chraibi M, Löhner R, Haug E, Gawenat B (2014) Qualitative validation of PEDFLOW for description of unidirectional pedestrian dynamics; the conference in pedestrian and evacuation dynamics 2014 (PED2014). Transp Res Proc 2:733-738

116. Zhang Y, Feng G, Kang Z, Bi Y, Cai Y (2017) Numerical simulation of coughed droplets in conference room. In: 10th international symposium on heating, ventilation and air conditioning, ISHVAC2017, October, 19-22 Jinan, China. Proc Eng 205:302-308
117. Zohdi TI (2020) Rapid simulation of viral decontamination efficacy with UV irradiation. Comput Methods Appl Mech Eng. https://doi.org/10.1016/j.cma.2020.113216

118. Zohdi TI (2020) Modeling and simulation of the infection zone from a cough. Comput Mech. https://doi.org/10.1007/ s00466-020-01875-5

Publisher's Note Springer Nature remains neutral with regard to jurisdictional claims in published maps and institutional affiliations. 